\title{
Greening of the brown-dwarf desert
}

\section{EPIC 212036875b: a $51 M_{\mathrm{J}}$ object in a 5-day orbit around an F7 V star $\star, \star \star$}

\author{
Carina M. Persson ${ }^{1}$, Szilárd Csizmadia ${ }^{2}$, Alexander J. Mustill ${ }^{3}$, Malcolm Fridlund ${ }^{1,4}$, Artie P. Hatzes ${ }^{5}$, \\ Grzegorz Nowak $^{6,7}$, Iskra Georgieva ${ }^{1}$, Davide Gandolfi ${ }^{8}$, Melvyn B. Davies ${ }^{3}$, John H. Livingston ${ }^{9}$, Enric Palle ${ }^{6,7}$, \\ Pilar Montañes Rodríguez ${ }^{6,7}$, Michael Endl ${ }^{10}$, Teruyuki Hirano ${ }^{11}$, Jorge Prieto-Arranz ${ }^{6,7}$, Judith Korth ${ }^{12}$, \\ Sascha Grziwa ${ }^{12}$, Massimiliano Esposito ${ }^{5}$, Simon Albrecht ${ }^{13}$, Marshall C. Johnson ${ }^{14}$, Oscar Barragán ${ }^{8,15}$, \\ Hannu Parviainen ${ }^{6,7}$, Vincent Van Eylen ${ }^{16}$, Roi Alonso Sobrino ${ }^{6,7}$, Paul G. Beck ${ }^{6,7,17}$, Juan Cabrera ${ }^{2}$, \\ Ilaria Carleo ${ }^{18}$, William D. Cochran ${ }^{10}$, Fei Dai ${ }^{16,19}$, Hans J. Deeg ${ }^{6,7}$, Jerome P. de Leon ${ }^{9}$, Philipp Eigmüller ${ }^{2}$, \\ Anders Erikson $^{2}$, Akai Fukui ${ }^{20}$, Lucía González-Cuesta ${ }^{6,7}$, Eike W. Guenther ${ }^{5}$, Diego Hidalgo ${ }^{6,7}$, Maria Hjorth ${ }^{13}$, \\ Petr Kabath $^{21}$, Emil Knudstrup ${ }^{13}$, Nobuhiko Kusakabe ${ }^{20,22}$, Kristine W. F. Lam ${ }^{23}$, Mikkel N. Lund ${ }^{13}$, \\ Rafael Luque $^{6,7}$, Savita Mathur ${ }^{6,7}$, Felipe Murgas ${ }^{6,7}$, Norio Narita ${ }^{6,9,20,22,24}$, David Nespral ${ }^{6,7}$, Prajwal Niraula ${ }^{25}$, \\ A. O. Henrik Olofsson ${ }^{1}$, Martin Pätzold ${ }^{12}$, Heike Rauer ${ }^{2,23}$, Seth Redfield ${ }^{18}$, Ignasi Ribas ${ }^{26,27}$, \\ Marek Skarka ${ }^{21,28}$, Alexis M. S. Smith ${ }^{2}$, Jan Subjak ${ }^{21,29}$, and Motohide Tamura ${ }^{9,20,22}$
}

(Affiliations can be found after the references)

Received 21 March 2019 / Accepted 13 June 2019

\begin{abstract}
Context. Although more than 2000 brown dwarfs have been detected to date, mainly from direct imaging, their characterisation is difficult due to their faintness and model-dependent results. In the case of transiting brown dwarfs, however, it is possible to make direct high-precision observations.

Aims. Our aim is to investigate the nature and formation of brown dwarfs by adding a new well-characterised object, in terms of its mass, radius and bulk density, to the currently small sample of less than 20 transiting brown dwarfs.

Methods. One brown dwarf candidate was found by the KESPRINT consortium when searching for exoplanets in the K2 space mission Campaign 16 field. We combined the K2 photometric data with a series of multicolour photometric observations, imaging, and radial velocity measurements to rule out false positive scenarios and to determine the fundamental properties of the system.

Results. We report the discovery and characterisation of a transiting brown dwarf in a 5.17-day eccentric orbit around the slightly evolved F7 V star EPIC 212036875. We find a stellar mass of $1.15 \pm 0.08 M_{\odot}$, a stellar radius of $1.41 \pm 0.05 R_{\odot}$, and an age of $5.1 \pm 0.9$ Gyr. The mass and radius of the companion brown dwarf are $51 \pm 2 M_{\mathrm{J}}$ and $0.83 \pm 0.03 R_{\mathrm{J}}$, respectively, corresponding to a mean density of $108_{-13}^{+15} \mathrm{~g} \mathrm{~cm}^{-3}$.

Conclusions. EPIC $212036875 \mathrm{~b}$ is a rare object that resides in the brown-dwarf desert. In the mass-density diagram for planets, brown dwarfs, and stars, we find that all giant planets and brown dwarfs follow the same trend from $\sim 0.3 M_{\mathrm{J}}$ to the turn-over to hydrogen burning stars at $\sim 73 M_{\mathrm{J}}$. EPIC $212036875 \mathrm{~b}$ falls close to the theoretical model for mature $\mathrm{H} / \mathrm{He}$ dominated objects in this diagram as determined by interior structure models. We argue that EPIC $212036875 \mathrm{~b}$ formed via gravitational disc instabilities in the outer part of the disc, followed by a quick migration. Orbital tidal circularisation may have started early in its history for a brief period when the brown dwarf's radius was larger. The lack of spin-orbit synchronisation points to a weak stellar dissipation parameter $\left(Q_{\star}^{\prime} \gtrsim 10^{8}\right)$, which implies a circularisation timescale of $\gtrsim 23 \mathrm{Gyr}$, or suggests an interaction between the magnetic and tidal forces of the star and the brown dwarf.
\end{abstract}

Key words. planetary systems - stars: fundamental parameters - stars: individual: EPIC 212036875 - techniques: photometric techniques: radial velocities

\section{Introduction}

The dividing line between gaseous giant planets (GPs) and brown dwarfs (BDs) is still unclear, largely due to the lack

\footnotetext{
* Table 2 is only available at the CDS via anonymous ftp to cdsarc.u-strasbg.fr (130.79.128.5) or via http://cdsarc. u-strasbg.fr/viz-bin/qcat?]/A+A/628/A64

$\star \star$ This work is done under the framework of the KESPRINT collaboration (http://kesprint.science). KESPRINT is an international consortium devoted to the characterisation and research of exoplanets discovered with space-based missions.
}

of well-characterised objects in this mass range. BDs have classically been regarded as objects between large planets and low-mass stars. Their masses have been defined as being in the range $13-80 M_{\mathrm{J}}$ (Burrows et al. 2001), which sustains deuterium burning through nuclear fusion for typically 0.1 million years, but below the ignition limit of hydrogen at $75-80 M_{\mathrm{J}}$. Objects with masses above $65 M_{\mathrm{J}}$ also burn lithium. The exact limits depend on models and internal chemical composition (Dieterich et al. 2014; Spiegel et al. 2011; Baraffe et al. 2002). Another division between GPs and BDs is based on formation. BDs are considered to form like stars from gravitational 
instability on a dynamical timescale with the elemental abundance of the interstellar medium, while GPs form on a longer timescale by core accretion with an enhanced metal abundance compared to the host star (Chabrier et al. 2014). By this definition, the mass domains overlap since the minimum BD mass is about $3 M_{\mathrm{J}}$, and the maximum planet mass can be as high as tens of $M_{\mathrm{J}}$. Others argue that BDs should not be distinguished from hydrogen-burning stars as they have more similarities to stars than planets (Whitworth 2018). Hatzes \& Rauer (2015), on the other hand, suggest that BDs should be classified as GPs instead of a separate class of their own based on the massdensity relationship. They defined objects within a mass range of $\sim 0.3-60 M_{\mathrm{J}}$ as the gaseous planet sequence, in analogy with the main sequence of stars. Objects below and above these limits were considered to be low-mass planets and low-mass stars, respectively, although the upper limit could be as high as $80 M_{\mathrm{J}}$. This was corroborated by Chen \& Kipping (2017) who found that BDs follow the same trend as GPs in the mass-radius diagram up to $80 M_{\mathrm{J}}$.

Although more than 2000 BDs have been detected (e.g. Skrzypek et al. 2016; Johnston 2015) mainly by large-scale direct imaging surveys, most of the detected BDs are free-floating, and only about 400 are found in bound systems at large distances from the primary star. Close BD companions to a main sequence star are very rare. Several surveys have showed that BDs in close orbits ( $<3 \mathrm{AU})$ around main sequence FGKM stars have a much lower frequency than GPs and close binaries (e.g. Marcy \& Butler 2000; Grether \& Lineweaver 2006; Sahlmann et al. 2011). This is commonly referred to as the $\mathrm{BD}$ desert, and may be a consequence of different formation mechanisms for low- and high-mass BDs. BDs with masses $35 \lesssim M \sin i \lesssim 55 M_{\mathrm{J}}$ and orbital periods shorter than 100 days may represent the driest part of this desert (Ma \& Ge 2014). For objects in very close orbits, $a<0.2 \mathrm{AU}$, Triaud et al. (2017) found a paucity of lower masses, 3-13 $M_{\mathrm{J}}$.

It is evident that many more well-characterised BDs are required to solve these issues. Characterisation from imaging is difficult since the objects are very faint, unless they are very young, and is heavily dependent on evolutionary models. In the case of eclipsing BDs the situation is different since accurate determination of diameters is possible with photometric observations of the host star. Mass measurements are also relatively easy to perform with high precision due to the high masses of BDs. It is therefore possible to perform a model-independent characterisation of individual BDs found by transit surveys combined with follow-up radial velocity (RV) measurements.

Space-based photometry allows excellent photometric precision and long uninterrupted observations (Fridlund 2018; Deleuil \& Fridlund 2018; Borucki 2018). This technique has successfully been used to detect thousands of transiting exoplanets by the space missions CoRoT, and Kepler and its extension $\mathrm{K} 2$. The recently launched TESS mission is expected to increase this number even further. The first discovery of a transiting BD, CoRoT-3b (Deleuil et al. 2008), was in fact made from space. The BD sample has since grown with additional detections from space, and also with the ground-based surveys SuperWASP, HATNet, MEarth, and KELT. Even so, the sample of wellcharacterised objects with masses between $\sim 10$ and $80 M_{\mathrm{J}}$ in bound systems is still very small. Many more are needed to investigate possible differences between GPs and BDs. Using the classic $13 M_{\mathrm{J}}$ limit between GPs and BDs, only 17 transiting BDs in bound systems around main sequence stars are known today. A summary of 11 BDs, five candidates, and two eclipsing BD binaries is found in Table III.6.1 of Csizmadia (2016).
Table 1. Basic parameters for EPIC $212036875^{(a)}$

\begin{tabular}{|c|c|}
\hline Parameter & Value \\
\hline \multicolumn{2}{|l|}{ Main Identifiers } \\
\hline EPIC & 212036875 \\
\hline 2MASS & J08584567+2052088 \\
\hline WISE & J085845.66+205208.4 \\
\hline TYC & $1400-1873-1$ \\
\hline UCAC & $555-045746$ \\
\hline Gaia DR2 & 684893489523382144 \\
\hline \multicolumn{2}{|l|}{ Equatorial coordinates } \\
\hline$\alpha(\mathrm{J} 2000.0)$ & $08^{\mathrm{h}} 58^{\mathrm{m}} 45.67$ \\
\hline$\delta(\mathrm{J} 2000.0)$ & $+20^{\circ} 52^{\prime} 08^{\prime \prime} .78$ \\
\hline \multicolumn{2}{|l|}{ Magnitudes } \\
\hline$B$ (Johnson) & $11.654 \pm 0.113$ \\
\hline$V$ (Johnson) & $10.950 \pm 0.095$ \\
\hline$G($ Gaia $)$ & $10.9148 \pm 0.0009$ \\
\hline Kepler & 10.937 \\
\hline$g$ & $12.257 \pm 0.050$ \\
\hline$r$ & $10.918 \pm 0.060$ \\
\hline$i$ & $10.800 \pm 0.070$ \\
\hline$J$ & $10.042 \pm 0.022$ \\
\hline$H$ & $9.843 \pm 0.024$ \\
\hline$K$ & $9.774 \pm 0.018$ \\
\hline Parallax (mas) & $3.238 \pm 0.048$ \\
\hline Systemic velocity $\left(\mathrm{km} \mathrm{s}^{-1}\right)$ & $-22.7 \pm 1.7$ \\
\hline$\mu_{R A}\left({\left.\operatorname{mas~} \mathrm{yr}^{-1}\right)}\right.$ & $-2.62 \pm 0.08$ \\
\hline$\mu_{D e c}\left(\operatorname{mas~yr}^{-1}\right)$ & $-29.70 \pm 0.05$ \\
\hline
\end{tabular}

Notes. ${ }^{(a)}$ From the Ecliptic Plane Input Catalogue (EPIC; Huber et al. 2016) http://archive.stsci.edu/k2/epic/search.php and the Gaia DR2 archive http://gea.esac.esa.int/archive/

Later discoveries of six additional BDs have been made from space: Kepler-503b (Cañas et al. 2018), EPIC 219388192b (Nowak et al. 2017), EPIC 201702477b (Bayliss et al. 2017), and from the ground: WASP-128b (Hodžić et al. 2018), LP 261-75b (Irwin et al. 2018), and HATS-70b (Zhou et al. 2019).

In this paper we report the independent discovery and observations of EPIC $212036875 \mathrm{~b}$ performed by the KESPRINT consortium (e.g. Hjorth et al. 2019; Korth et al. 2019; Livingston et al. 2019; Palle et al. 2019; Gandolfi et al. 2018). EPIC $212036875 \mathrm{~b}$ was found in the K2 Campaign 16, and follow-up observations subsequently revealed that the object was the 18th transiting $\mathrm{BD}$ detected to date. We note that shortly before submitting this article, Carmichael et al. (2019) publicly announced their discovery and RV observations of EPIC 212036875 b. We describe the K2 photometry in Sect. 2 and the follow-up observations in Sect. 3. We model the star in Sect. 4, and the transit and RVs in Sect. 5. We end the paper with a discussion and conclusions in Sect. 6 and 7, respectively.

\section{K2 photometry and transit detection}

Between 7 Dec. 2017 and 25 Feb. 2018, the Kepler space telescope monitored 35643 objects in the long cadence mode (29.4 $\mathrm{min})$, and 131 objects with short cadence ( $1 \mathrm{~min}$ ) in the direction towards (J2000) $\alpha=08^{\mathrm{h}} 54^{\mathrm{m}} 50^{\prime} \cdot 3$ and $\delta=+01^{\circ} 14^{\prime} 06^{\prime \prime} 0$ (the K2 Campaign $16^{1}$ ). The data of

\footnotetext{
1 https://keplerscience.arc.nasa.gov/

k2-data-release-notes.html\#k2-campaign-16
} 


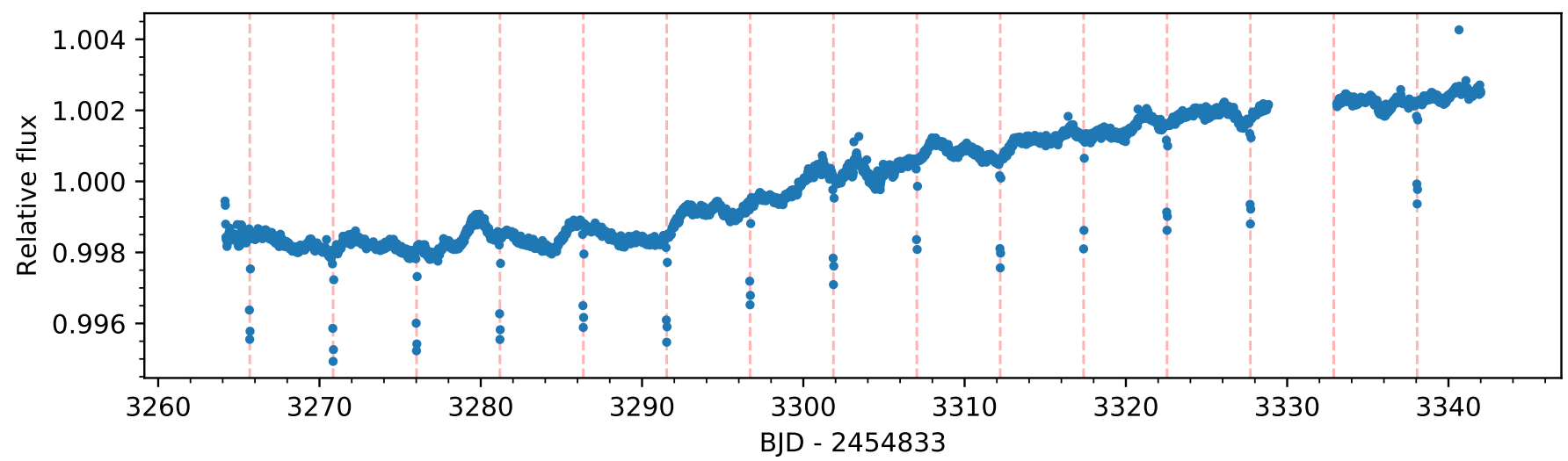

Fig. 1. Pre-processed Vanderburg K2 light curve of EPIC 212036875. The dashed vertical lines mark the 14 narrow and shallow brown dwarf transits used in the analysis. We also mark a missing transit located in a gap of the light curve. The broader periodic variation of approximately $0.07 \%$ is caused by stellar activity.

Campaign 16 was downloaded from the Mikulski Archive for Space Telescopes ${ }^{2}$ (MAST). We followed the procedure described in Korth et al. (2019) and searched for periodic signals in the photometric data using the EXOTRANS software (Grziwa et al. 2012). The software utilises wavelet-based filters to remove stellar variability and instrument systematics, and a modified box-fitting least squares (BLS; Kovács et al. 2002) algorithm, improved by implementing optimal frequency sampling (Ofir 2014), to detect the most significant transits. Periodic signals were detected in the light curve of the F7 V star EPIC 212036875 with an orbital period of 5.17 days, a midtransit time $T_{0}=3265.68$ days (BJD -2454833 ), and a depth of $\sim 0.4 \%$. The pre-processed Vanderburg ${ }^{3}$ light curve is shown in Fig. 1. The depth is consistent with a Jupiter-sized planet, although the nature of the planet candidate had to await radial velocity follow-up. We found no signs of even-odd depth variations or a secondary eclipse within $1 \sigma$, which is the first step to excluding binaries. We thus proceeded with a follow-up campaign to characterise the EPIC 212036875 system.

The basic parameters of the star are listed in Table 1.

\section{Ground-based follow-up}

We performed a series of follow-up observations with (i) multicolour photometric observations to rule out eclipsing binary false-positives (Sect. 3.1); (ii) reconnaissance spectra observations to remove candidates with rapidly rotating stars, double-lined binaries, and blends of spectral components (Sect. 3.2); (iii) RV follow-up to obtain the BD mass and coadded spectra needed for stellar spectral modelling (Sect. 3.3); and (iv) high-resolution adaptive optics (AO; Sect. 3.4) and speckle imaging (Sect. 3.5) to search for contaminant stars that may be background or foreground stars, or physically bounded eclipsing binaries whose light may be diluted by the target star thus generating transit-like signals. Speckle and AO observations are fundamentally different techniques. NESSI speckle probes the inner region $\left(<0.2^{\prime \prime}\right)$ around the target star at optical wavelengths, while AO achieves a much higher contrast in the $0.2^{\prime \prime}-1.0^{\prime \prime}$ region in the near-infrared. These regions are not possible to explore with the K2 data with a sky-projected pixel size of $4^{\prime \prime}$.

\footnotetext{
2 https://archive.stsci.edu/prepds/k2sff/

3 https://www. cfa.harvard. edu/ avanderb/k2c16/ ep212036875.html
}

\subsection{MUSCAT2}

We observed a full transit of EPIC 212036875 b with MuSCAT2 at the Carlos Sanchez Telescope (TCS) on the night of 3 April 2018. MuSCAT2 is a four-colour imager that allows simultaneous observations in $g^{\prime}, r^{\prime}, i^{\prime}$, and $z^{\prime}$ (Narita et al. 2019). The observations started at 20:15 UT and ended at 23:30 UT, covering the full transit and some pre- and post-transit baselines. The night was clear, with variable seeing between $1^{\prime \prime}$ and $2^{\prime \prime}$. Exposure times were set to $5 \mathrm{~s}$ in all channels.

The differential photometry and transit light curve analysis were carried out with a dedicated MuSCAT2 pipeline. The photometry followed standard aperture photometry practices: we calculated an astrometric solution for each frame using an offline version of astrometry.net (Lang et al. 2010), and retrieved the photometry for a set of comparison stars and aperture sizes.

The transit modelling continued by first choosing a set of optimal apertures that minimise the relative light curve pointto-point scatter. Next, we jointly fitted a transit model with a linear baseline model (a linear model in sky level, airmass, seeing, and CCD position variations) to the four light curves using PyTransit and LDTk (Parviainen 2015; Parviainen \& Aigrain 2015). Finally, we swapped the linear baseline model to a Gaussian process-based model with the final kernel consisting of a product of squared exponential kernels for all the covariates, and carried out MCMC sampling to obtain an estimate of the model parameter posterior distribution. The final light curves are shown in Fig. 2 and the data is listed in Table 2 (only available at the CDS). The transit model allows for colour-dependent variations in transit depth due to blending by an unresolved source, and our analysis allows us to rule out any significant contamination that would affect the parameter estimates derived from the transit photometry.

\subsection{Reconnaissance spectra with Tull}

On 5 April 2018 we obtained a reconnaissance spectrum of EPIC 212036875 with the Tull spectrograph at the $2.7 \mathrm{~m}$ telescope at McDonald Observatory. The highresolution $(R \approx 60000)$ spectrum was reduced using standard iraf routines. We derived a first estimate of the stellar spectroscopic parameters using the code Kea (Endl \& Cochran 2016): $T_{\text {eff }}=6380 \pm 58 \mathrm{~K},[\mathrm{Fe} / \mathrm{H}]=-0.21 \pm 0.03 \mathrm{dex}$, $\log \left(g_{\star}\right)=4.25 \pm 0.14(\mathrm{cgs})$, and $V \sin i_{\star}=11.9 \pm 0.3 \mathrm{~km} \mathrm{~s}^{-1}$. We found no evidence of a double-lined binary or any blends of spectral components. 

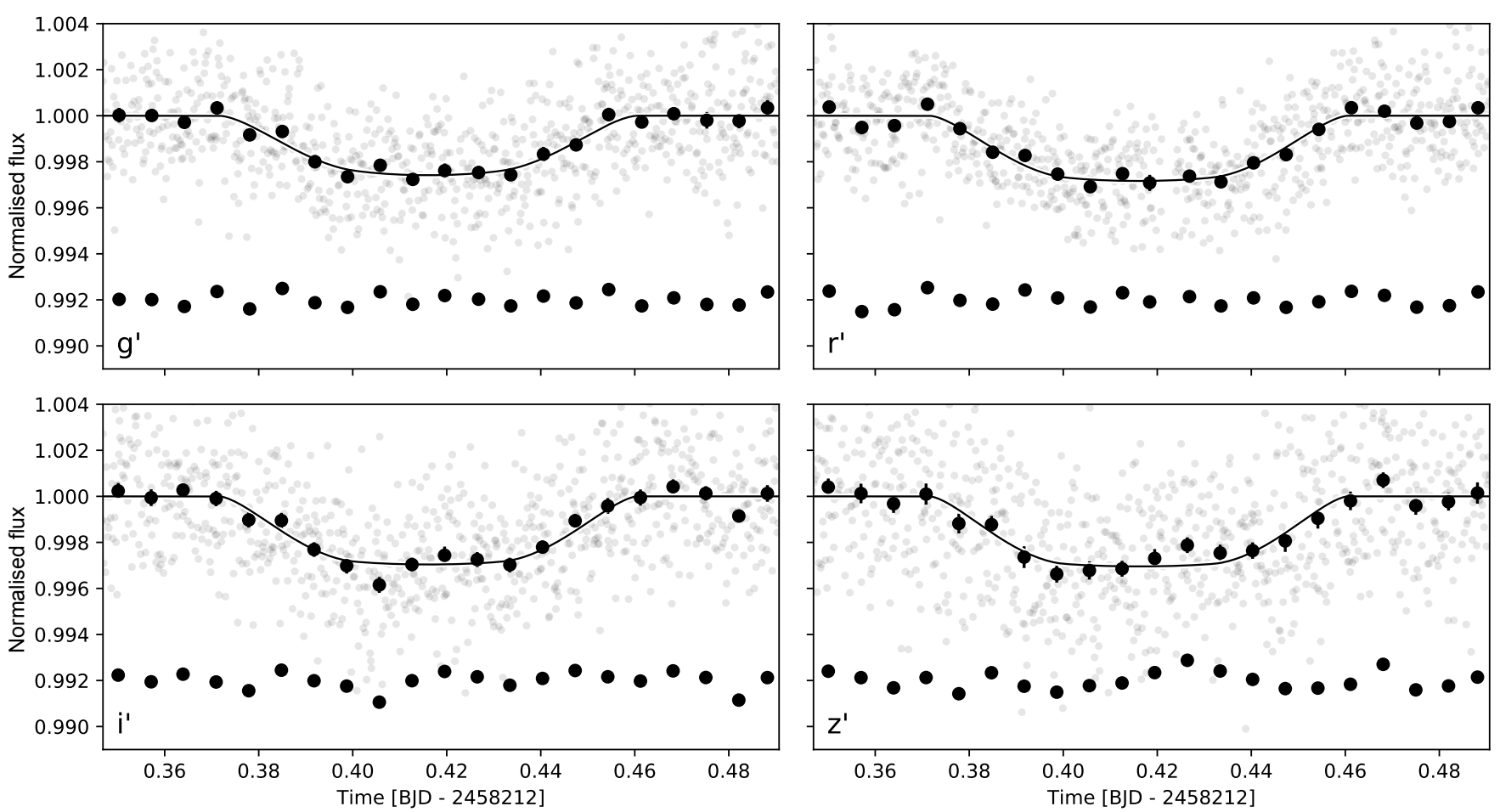

Fig. 2. Transit light curves of EPIC $212036875 \mathrm{~b}$ obtained with MuSCAT2 (TCS) in $g^{\prime}$ (upper left), $r^{\prime}$ (upper right), $i^{\prime}$ (lower left), and $z^{\prime}$ (lower right) filters. The black solid line is the best-fit model accounting for the de-trending and transit components. The residuals are plotted in the lower portion of each plot.

\subsection{Radial velocity follow-up with FIES}

The RV follow-up was performed with the FIbre-fed Échelle Spectrograph (FIES; Telting et al. 2014; Frandsen \& Lindberg 1999) mounted on the $2.56 \mathrm{~m}$ Nordic Optical Telescope (NOT) at the Roque de los Muchachos Observatory. We observed nine high-resolution $(R \approx 67000)$ spectra between 9 April and 22 May 2018 as part of our CAT and TAC programmes 57-015, 57-206, and 57-210, and OPTICON programme 2018A-044. To account for the RV offset caused by a major instrument refurbishment that occurred on 30 April 2018, we treated the RV taken between 9 and 26 April, and between 6 and 8 May as two independent data sets. In addition, 14 intermediate-resolution $(R \approx 47000)$ FIES spectra were also acquired between 12 May 2018 and 26 Feb. 2019, as part of the OPTICON programme 2018B-052 and the Spanish-Nordic programme 58-301. Depending on the sky conditions and scheduling constraints, we set the exposure times to 1800-3600 s for both resolutions. To trace the RV drift of the instrument we followed the strategy outlined in Gandolfi et al. (2015) and Buchhave et al. (2010) and bracketed the science exposures with long-exposed (60-90 s) ThAr spectra. We used the standard IRAF and IDL routines to reduce the data. The $\mathrm{S} / \mathrm{N}$ of the extracted spectra ranges between $\sim 35$ and 75 per pixel at $5500 \AA$ A. Radial velocities were extracted via multi-order cross-correlations with the spectrum of the RV standard star HD 168009, for which we adopted an absolute RV of $-64.650 \mathrm{~km} \mathrm{~s}^{-1}$ (Udry et al. 1999).

The FIES RVs are listed in Table A.1. Figure A.2 shows the generalised Lomb-Scargle periodogram of the offset-corrected Doppler measurements (combined by subtracting the systemic velocities listed in Table 5). We found a very significant peak at the orbital frequency of the transiting BD with a false alarm probability FAP $\ll 10^{-6}$, proving that the Doppler reflex motion of the star induced by the orbiting companion is clearly detected in our data.

\subsection{Subaru/IRCS AO imaging}

In order to obtain high-contrast, high-resolution images of EPIC 212036875, we performed AO imaging with the InfraRed Camera and Spectrograph (IRCS, Kobayashi et al. 2000) mounted on the Subaru $8.2 \mathrm{~m}$ telescope on 14 June 2018. The target star was used as a natural guide and AO correction was applied to obtain high-contrast $K^{\prime}$-band images of the target. We used the fine-sampling mode ( 1 pix $\approx 21$ mas), and implemented a five-point dithering to minimise the impacts of bad pixels and cosmic rays.

We reduced the raw frames with a standard procedure described in Hirano et al. (2016) to produce an aligned and combined image of EPIC 212036875. The full width at the half maximum of the co-added target image was 0.'089 suggesting that the AO correction worked well for this target. As shown in the inset of Fig. 3, EPIC 212036875 exhibits no nearby source. Following Hirano et al. (2018) we estimated the detection limit of possible nearby sources by computing a $5 \sigma$ contrast curve drawn in Fig. 3. The achieved contrast is $\Delta m_{K^{\prime}}>7 \mathrm{mag}$ beyond $0 .{ }^{\prime} 5$ from EPIC 212036875.

\subsection{NESSI imaging}

To further constrain the presence of stellar companions at close separations, we conducted speckle imaging of EPIC 212036875 using the NASA Exoplanet Star and Speckle Imager (NESSI; Scott \& Howell 2018) at the WIYN 3.5 m telescope on 19 June 2018 (programme ID 2018A-0181). NESSI operates simultaneously in two bands centred at $562 \mathrm{~nm}$ 


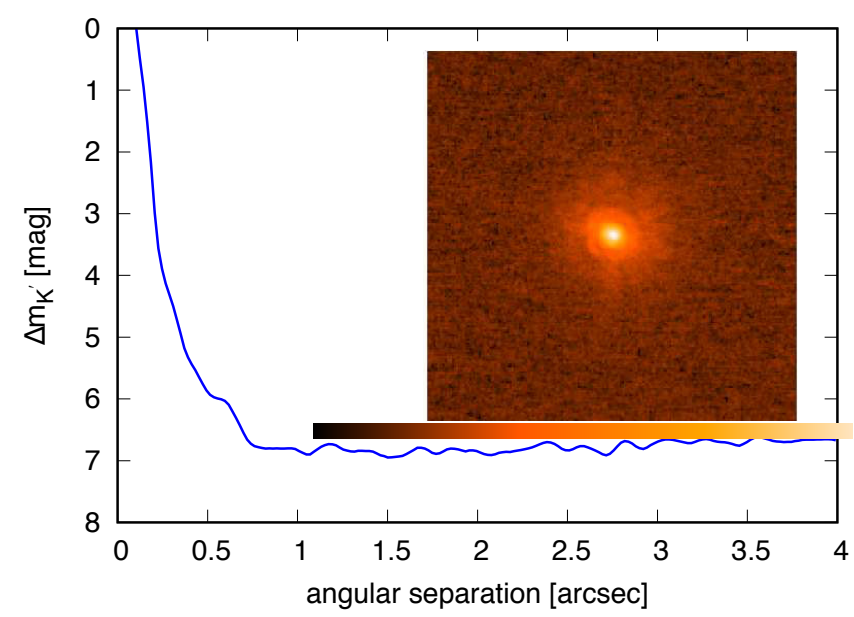

Fig. 3. IRCS/Subaru AO-imaging in the $K^{\prime}$-band and $5 \sigma$ magnitude contrast curve as a function of angular separation from EPIC 212036875. The inset shows the $16^{\prime \prime} \times 16^{\prime \prime}$ saturated image. North-east is up and to the left.

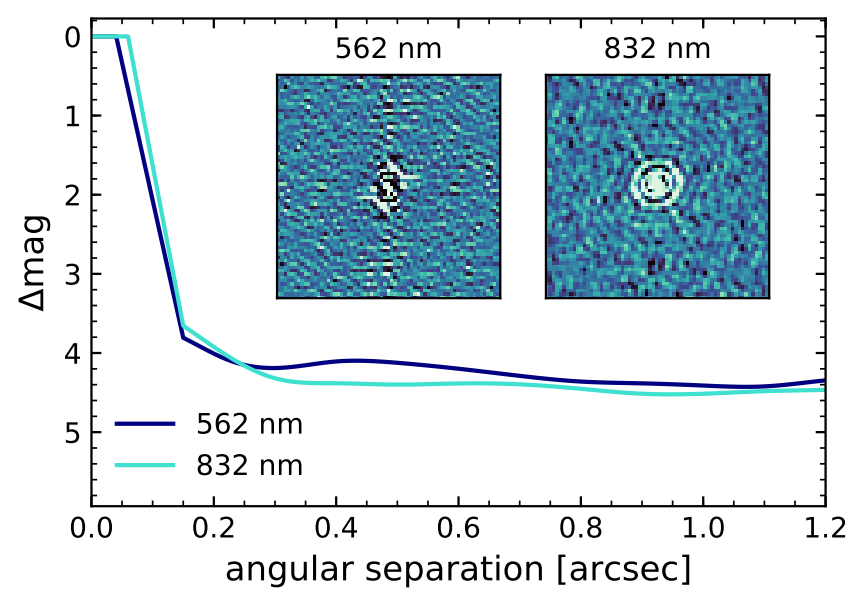

Fig. 4. NESSI/WIYN speckle interferometry reconstructed images in the $r$ - and $z$-narrowbands and $5 \sigma$ contrast curves. The inset images are $1^{\prime \prime} 2 \times 1$.' 2 . North-east is up and to the left.

( $r$-narrowband) and $832 \mathrm{~nm}$ ( $z$-narrowband). We collected and reduced the data following the procedures described by Howell et al. (2011), yielding 4". $6 \times 4$ "' 6 reconstructed images of the host star (the inset shows the central $11^{\prime \prime} 2 \times 1$ 1.'2 in Fig. 4). We did not detect any secondary sources in the reconstructed images. The $5 \sigma$ detection limits are shown in Fig. 4; the contrast is approximately 4.5 mag beyond 0.3 from EPIC 212036875 in both images.

We used the NESSI and Subaru magnitude limits to estimate limits on companion masses versus separation, and find that massive companions are excluded outside $\sim 100$ AU (Fig. A.1).

\section{Stellar analysis}

\subsection{Spectral analysis}

Before we modelled the BD, we first computed the absolute mass and radius of the host star. In order to obtain the stellar parameters needed in the stellar models, we used the spectral analysis package Spectroscopy Made Easy (SME; Valenti \& Piskunov 1996; Piskunov \& Valenti 2017). This software calculates synthetic stellar spectra from grids of atmosphere models which are then fitted to the observations using a $\chi^{2}$-minimising procedure. Here we specifically used the ATLAS 12 model spectra (Kurucz 2013), and SME version 5.22 to model our co-added FIES spectra. We followed well-established methods described in Fridlund et al. (2017) and Persson et al. (2018) to compute $T_{\text {eff }}, \log \left(g_{\star}\right)$, $V \sin i_{\star}$, and abundances. The micro- and macroturbulent velocities, $V_{\text {mic }}$ and $V_{\text {mac }}$, were fixed using the calibration equations for Sun-like stars from Bruntt et al. (2010) and Doyle et al. (2014), respectively. The line lists were taken from the Vienna Atomic Line Database ${ }^{4}$ (Ryabchikova et al. 2015).

Our results obtained with SME $\left(T_{\text {eff }}=6230 \pm 90 \mathrm{~K}\right)$ are in agreement with the values listed in the Gaia DR2 archive $\left(T_{\text {eff }}=6227 \pm 100 \mathrm{~K}\right)$ and EPIC $\left(T_{\text {eff }}=6336 \mathrm{~K}\right)$, and are also consistent with the Kea results from the Tull reconnaissance spectra in Sect. $3.2\left(T_{\text {eff }}=6380 \pm 58 \mathrm{~K}\right)$. The resulting $T_{\text {eff }}$ and the luminosity in the Gaia DR2 archive implies a spectral type of F7 V. All final results are listed in Table 3.

\subsection{Stellar mass and radius}

We used the Southworth (2011) calibration equations to compute the stellar mass and radius. These empirical relations, based on data from eclipsing binaries, are valid for masses up to $3 M_{\odot}$ and account for metal abundance and evolution. It provides the advantage of using the stellar density which has a higher precision than $\log \left(g_{\star}\right)$ since it is derived from the transit light curve. Additional input parameters are $T_{\text {eff }}$ and $[\mathrm{Fe} / \mathrm{H}]$.

We also compared the Southworth results with several other, independent methods. The first is the Torres et al. (2010) calibration equations based on a different set of eclipsing binaries, as well as interferometrically determined stellar diameters. The input parameters are $T_{\text {eff }}, \log \left(g_{\star}\right)$, and $[\mathrm{Fe} / \mathrm{H}]$. We also applied the Bayesian PARAM $1.3^{5}$ model tool tracks (da Silva et al. 2006) with the PARSEC isochrones (Bressan et al. 2012) and the apparent visual magnitude, $T_{\text {eff }},[\mathrm{Fe} / \mathrm{H}]$, and the parallax as input. The derived age and $\log \left(g_{\star}\right)$ from PARAM 1.3 are $5.1 \pm 0.9 \mathrm{Gyr}$ and $4.10 \pm 0.04(\mathrm{cgs})$, respectively. Finally, when we compared the derived mass and radius to a typical F7 V star, we noted that EPIC 212036875 seems to be slightly evolved, in line with a typical life time of about $\sim 7$ Gyr. EPIC 212036875 b is one of only two BDs where the age can be determined relatively precisely, due to its evolutionary state. The other BD with a welldetermined age is EPIC 219388192b (Nowak et al. 2017), which is a member of Ruprecht 147, the oldest nearby open cluster association.

All models are in excellent agreement with each other. The results from all models are listed in Table 4, and the final adopted stellar parameters are listed in Table 3.

\subsection{Stellar rotation period}

The K2 light curve of EPIC 212036875 displays periodic and quasi-periodic photometric variations with a semi-amplitude of $\sim 0.07 \%$. These are superimposed on a long-term photometric trend with a peak-to-peak amplitude of $\sim 0.4 \%$ (Fig. 1), which we attributed to the slow drift often present in K2 data (Vanderburg \& Johnson 2014). Given the spectral type of the host star, the periodic and quasi-periodic variability is likely induced by magnetically active regions carried around by stellar rotation.

We used the generalised Lomb-Scargle (GLS) periodogram (Zechmeister \& Kürster 2009) and the auto-correlation function

\footnotetext{
4 http://vald.astro.uu.se

5 http://stev.oapd.inaf.it/cgi-bin/param_1.3
} 
Table 3. Adopted stellar parameters of EPIC 212036875.

\begin{tabular}{ll}
\hline \hline Parameter & EPIC 212036875 \\
\hline Effective temperature $T_{\mathrm{eff}}{ }^{(a)}(\mathrm{K})$ & $6230 \pm 90$ \\
Surface gravity $\log \left(g_{\star}\right)^{(a),(b)}(\mathrm{cgs})$ & $4.17 \pm 0.10$ \\
Metallicity [Fe/H] ${ }^{(a)}(\mathrm{dex})$ & $-0.28 \pm 0.05$ \\
Metallicity $[\mathrm{Ca} / \mathrm{H}]^{(a)}(\mathrm{dex})$ & $-0.14 \pm 0.05$ \\
Metallicity $[\mathrm{Na} / \mathrm{H}]^{(a)}(\mathrm{dex})$ & $-0.11 \pm 0.05$ \\
Metallicity $[\mathrm{Mg} / \mathrm{H}]^{(a)}(\mathrm{dex})$ & $-0.16 \pm 0.05$ \\
Rotation velocity $V$ sin $i_{\star}{ }^{(a),(c)}\left(\mathrm{km} \mathrm{s}^{-1}\right)$ & $10.8 \pm 1.5$ \\
Microturbulent $V^{(d)}\left(\mathrm{km} \mathrm{s}^{-1}\right)$ & 1.3 \\
Macroturbulent $V^{(e)}\left(\mathrm{km} \mathrm{s}^{-1}\right)$ & 5.2 \\
Mass $M_{\star}(f)\left(M_{\odot}\right)$ & $1.15 \pm 0.08$ \\
Radius $R_{\star}(f)\left(R_{\odot}\right)$ & $1.41 \pm 0.05$ \\
Density $\rho_{\star}{ }^{(g)}\left(\mathrm{g} \mathrm{cm}{ }^{-3}\right)$ & $0.55 \pm 0.04$ \\
Luminosity $L_{\star}{ }^{(h)}\left(L_{\odot}\right)$ & $3.01_{-0.07}^{+0.05}$ \\
Spectral type & $\mathrm{F} 7 \mathrm{~V}$ \\
Rotation period & $7.2 \pm 0.5$ \\
Age ${ }^{(j)}(\mathrm{Gyr})$ & $5.1 \pm 0.9$ \\
\hline
\end{tabular}

Notes. ${ }^{(a)}$ From SME modelling. ${ }^{(b)}$ Modelled using Mg I. The Ca I model gives $\log \left(g_{\star}\right)=4.20 \pm 0.20$ (cgs). ${ }^{(c)}$ The projected stellar rotation speed of its surface. ${ }^{(d)}$ Fixed with the empirical calibration by Bruntt et al. (2010). ${ }^{(e)}$ Fixed with the empirical calibration by Doyle et al. (2014). ${ }^{(f)}$ Southworth (2011) calibration equation. ${ }^{\left({ }^{g}\right)}$ Density from pyaneti transit modelling in Sect. 5. Density from adopted stellar mass and radius is $0.58 \pm 0.08 \mathrm{~g} \mathrm{~cm}^{-3} \cdot{ }^{(h)}$ Gaia DR2 archive. ${ }^{(i)}$ From the generalised Lomb-Scargle periodogram. ${ }^{(j)}$ PARAM 1.3.

Table 4. Stellar mass and radius of EPIC 212036875 as derived from different methods.

\begin{tabular}{lrr}
\hline \hline Method & $\begin{array}{r}M_{\star} \\
\left(M_{\odot}\right)\end{array}$ & $\begin{array}{r}R_{\star} \\
\left(R_{\odot}\right)\end{array}$ \\
\hline Southworth $^{(a)}$ & $1.15 \pm 0.08$ & $1.41 \pm 0.05$ \\
Torres $^{(b)}$ & $1.19 \pm 0.09$ & $1.43 \pm 0.29$ \\
PARAM 1.3 $_{\text {Faia DR2 }^{(c)}}$ & $1.10 \pm 0.04$ & $1.52 \pm 0.06$ \\
EPIC $^{(d)}$ & $\ldots$ & $1.49 \pm 0.05$ \\
Spectral type $^{(e)} \mathrm{F} 7 \mathrm{~V}$ & $1.21 \pm 0.11$ & $1.38_{-0.17}^{+0.32}$ \\
\hline
\end{tabular}

Notes. The typical values for a F7 V star are listed as comparison. ${ }^{(a)}$ Southworth (2011) calibration equations. ${ }^{(b)}$ Torres et al. (2010) calibration equations. ${ }^{(c)}$ Gaia DR2 archive. ${ }^{(d)}$ The K2 Ecliptic Plane Input Catalog. ${ }^{(e)}$ Cox (2000).

(ACF) method (McQuillan et al. 2014) to estimate the rotation period of the star. Prior to computing the GLS periodogram and the ACF, we masked out the transits and removed the long-term trend by dividing the out-of-transit light curve by the best-fitting fourth-order cubic spline (Fig. A.3, upper panel). The GLS periodogram of the corrected light curve (Fig. A.3, middle panel) shows a very significant peak at $f=0.14 \mathrm{~d}^{-1}$ $\left(P_{\text {rot }}=7.2\right.$ days $)$ with FAP $\ll 10^{-6}$, estimated from the bootstrap method (Kuerster et al. 1997). The ACF of the light curve (Fig. A.3, lower panel) shows correlation peaks at $\sim 7,14,21$, and 28 days. We interpreted the peak at $\sim 7$ days as the rotation period of the star and the peaks at $\sim 14,21$, and 28 days as its first, second, and third harmonics, respectively. By fitting a Gaussian function to the highest peak of the GLS periodogram, we derived a rotation period of $P_{\text {rot }}=7.2 \pm 0.5$ days. Assuming that the star is seen almost equator-on $\left(\sin i_{\star} \approx 1\right)$, the spectroscopically derived rotational velocity $V \sin i_{\star}$ and the stellar radius imply a rotation period of $6.6 \pm 0.9$ days, in very good agreement with our results. The orbital period of the BD is thus within $7 \%$ to a $3: 2$ commensurability with the stellar rotation period.

We used the formula from Winn et al. (2007) to constrain $i_{\star}$ (the inclination of the stellar spin axis relative to the sky plane), and found $\sin i_{\star}=V \sin i_{\star} P_{\text {rot }} /\left(2 \pi R_{\star}\right) \approx 1.09 \pm 0.17$. The value with $\sin i_{\star}>1$ was rejected as unphysical and we determined a lower bound of $i_{\star}$ to $66^{\circ}$ with $1 \sigma$ confidence.

Since the $V \sin i_{\star}$ of EPIC 212036875 is relatively high, the Rossiter-McLaughlin (RM) effect could be measured with state-of-the-art spectrographs, mounted on $8-10 \mathrm{~m}$ class telescopes, using either RV RM or Doppler tomographic methodology. A first-order estimate of the amplitude of the RM effect is $\sim 16 \mathrm{~m} \mathrm{~s}^{-1}$ using the equation $\Delta V=\left(R_{\mathrm{bd}} / R_{\star}\right)^{2} \times \sqrt{1-b^{2}} \times V \sin i_{\star}$ (Winn 2010; Triaud 2018). We note, however, that with a large impact parameter the actual amplitude of the RM effect is a strong function of the angle between the sky projections of the stellar spin axis and the orbit normal $(\lambda)$, implying that the actual RM amplitude could vary substantially from the above estimate.

Apart from the independent measurements of $V \sin i_{\star}$ and $\lambda$, the measurement of the RM effect, together with the $P_{\text {rot }}$ and $V \sin i_{\star}$ measurements to constrain the inclination of the stellar rotation axis, would also allow a constraint upon the misalignment angle, $\psi$ (the 3-D obliquity angle between the stellar spin axis and the orbital axis). Measuring the spin-orbit misalignment of EPIC 212036875 b would be valuable because there are only a handful of such measurements available for transiting BDs (Triaud et al. 2009, 2013; Siverd et al. 2012; Zhou et al. 2019). Furthermore, this object is the only one of these for which the full 3D spin-orbit angle is measurable, allowing better constraints on the system architecture. Finally, all of the other objects observed to date have circular orbits, unlike EPIC 212036875 b; measuring the spin-orbit misalignment will enable a full dynamical characterisation of this system, which will have consequences for our understanding of how the system formed (see Sect. 6.2).

\section{Transit and radial velocity modelling}

We used the well-tested and publicly available PYTHON/FORTRAN pyaneti ${ }^{6}$ package (Barragán et al. 2019) to carry out simultaneous modelling of the K2 light curve and the FIES RV measurements. The code uses Markov chain Monte Carlo (MCMC) methods based on Bayesian analysis, and we have used it successfully (e.g. Gandolfi et al. 2019 and Barragán et al. 2018a). In preparation for the modelling, the light curve was detrended with the exotrending (Barragán \& Gandolfi 2017) code. This procedure reduces the flux variations of any long-term systematic or instrumental trends. Each of the 14 transits was cut out of the light curve, and four hours around each transit were masked to ensure that no in-transit data was used in the process before fitting a second-order polynomial to the remaining out-of-transit data.

\footnotetext{
6 https://github.com/oscaribv/pyaneti
} 


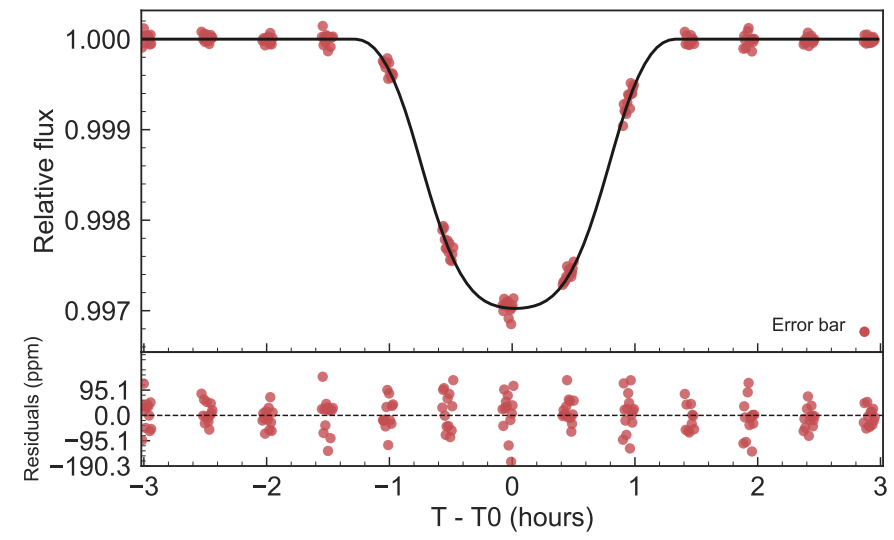

Fig. 5. Transit light curve folded to the orbital period of EPIC $212036875 \mathrm{~b}$. The K2 photometric data is indicated with the red points, and the best-fit transit model with the solid black line. The residuals of the fit are shown in the lower panel.

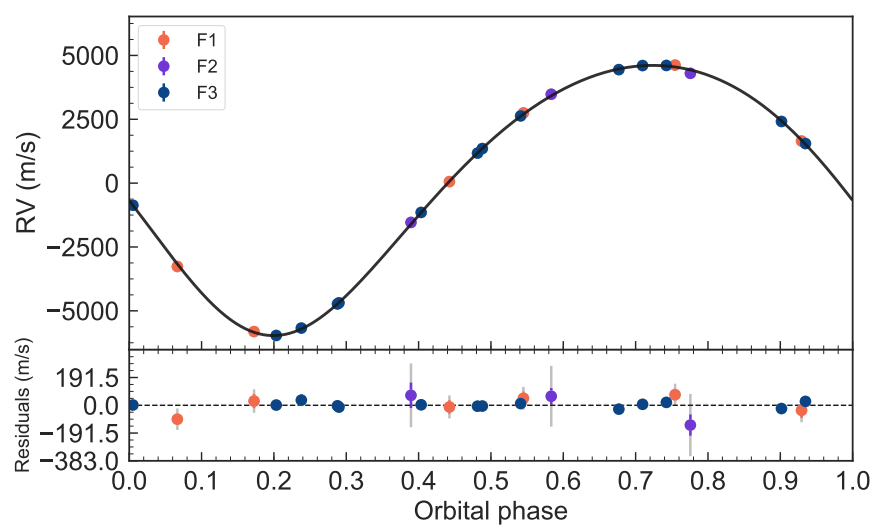

Fig. 6. Radial velocity curve of EPIC 212036875 phase folded to the orbital period of the brown dwarf. The different FIES set-ups are colourcoded (see inset), and the best-fit RV model is indicated with the solid black line. The residuals of the fit are shown in the lower panel. The coloured error bars are without jitter, and the grey error bars includes the jitter.

Following Barragán et al. (2018b), we fitted a Keplerian orbit to the RV data with an offset term for each systemic velocity from the different instrumental set-ups. We fitted for the scaled orbital distance $\left(a / R_{\star}\right)$, the eccentricity $(e)$, the argument of periastron $(\omega)$, the impact parameter $\left(b=a \cos (i) / R_{\star} \frac{1-e^{2}}{1+e \sin (\omega)}\right)$, the Doppler semi-amplitude variation $(K)$, the orbital period $\left(P_{\text {orb }}\right)$, the mid-transit time $\left(T_{0}\right)$, and the BD-to-star radius ratio $\left(R_{\mathrm{BD}} / R_{\star}\right)$. We used flat uniform priors over the ranges listed in Table 5, except for the limb-darkening coefficients (LDCs). Since the observational cadence of $\mathrm{K} 2$ is close to an integer fraction of the orbital period, the data points appear in clumps in the folded light curve in phase space, as shown in Fig. 5. The ingress and egress are not well sampled, and the LDCs are poorly constrained by the data. We therefore used Gaussian priors and the Mandel \& Agol (2002) quadratic limb-darkening equation based on the linear and quadratic coefficients $u_{1}$ and $u_{2}$, respectively. We used the Kipping (2013) parameterisation $q_{1}=\left(u_{1}+u_{2}\right)^{2}$ and $q_{2}=0.5 u_{1}\left(u_{1}+u_{2}\right)^{-1}$, and an interpolation 7 of the Claret \& Bloemen (2011) limb-darkening tables to our

\footnotetext{
7 http://astroutils.astronomy.ohio-state.edu/exofast/ limbdark. shtml (Eastman et al. 2013).
}

spectroscopic parameters and the Kepler bandpass to set Gaussian priors to $q_{1}$ and $q_{2}$. We used conservative 0.1 error bars on both the linear and quadratic coefficients.

To account for the long K2 integration time of almost $30 \mathrm{~min}$, we integrated the transit models over ten steps (Kipping 2010). The parameter space was explored with 500 independent chains randomly created inside the prior ranges. Convergence was checked after every 5000 iterations, and when reached the last 5000 iterations were used to create a posterior distribution of 250000 independent points for every parameter. We removed one outlier from the light curve. Since $\chi^{2} /$ d.o.f $=1.3$, we fitted for an RV jitter term for each instrument set-up in the model to take into account additional instrumental noise not included in the uncertainties and stellar activity-induced variation, and a light curve jitter term to account for the dispersion of the in- and out-of-transit data to obtain $\chi^{2} /$ d.o.f $=1.0$.

The high RV amplitude of about $5 \mathrm{~km} \mathrm{~s}^{-1}$ in Fig. 6 immediately signalled that the mass of the transiting object is much higher than the mass expected from a Jupiter-like planet. This is not possible to derive from the light curve alone since BDs and Jupiters have approximately the same size. The final mass is about $5 \%$ of the stellar host mass. We also note that the BD is near grazing as the derived impact parameter is $0.920_{-0.006}^{+0.005}$, which suggests that the derivation of limb darkening may be less accurate (Csizmadia et al. 2013). If EPIC 212036875 had a typical radius of an F7 V star instead of being slightly evolved (with about $8 \%$ larger radius), the BD would be grazing.

Carmichael et al. (2019) used the TRES spectrograph at the $1.5 \mathrm{~m}$ Tillinghast telescope at Mt. Hopkins, Arizona, with a spectral resolution $R=44000$ covering 390-910 nm to measure 14 RVs with $S / N \approx 22-45$ of EPIC 212036875 . This can be compared to our $23 \mathrm{RVs}$ with $S / N \approx 35-75$. Their uncertainties are somewhat larger than ours, but our results agree within $1 \sigma$.

The final results are listed in Table 5. We used the median and $68.3 \%$ credible interval of the posterior distributions which were all smooth and unimodal. We show the folded light curve with the best-fit transit model in Fig. 5, and the phase-folded RV curve with our best-fit model in Fig. 6.

\section{Discussion}

EPIC $212036875 \mathrm{~b}$ is a rare type of object in the BD desert. In this section we investigate its formation and tidal circularisation; in addition, we make comparison of GPs and BDs in the massdensity diagram.

\subsection{Formation}

There are several different paths to BD formation (for a summary, see e.g. Whitworth 2018). Objects with masses that range from stellar-like down to about $3 M_{\mathrm{J}}$ can form through gravitational collapse and turbulent fragmentation, as stars do (Padoan \& Nordlund 2004; Hennebelle \& Chabrier 2008). In protoplanetary discs, BDs can form possibly up to a few tens of $M_{\mathrm{J}}$ according to the core-accretion planet formation theory, either via traditional planetesimal accretion or via later pebble accretion variants (e.g. Pollack et al. 1996; Rice \& Armitage 2003; Alibert et al. 2004; Lambrechts \& Johansen 2012; Mordasini et al. 2012). For EPIC 212036875 b with a mass of $51 \pm 2 M_{\mathrm{J}}$, too massive for formation by core accretion, formation by gravitational instability in the protoplanetary disc may instead be possible (Toomre 1964; Kratter \& Lodato 2016). Disc fragmentation typically occurs at radii $>10$ AU and forms fragments with initial masses of a few to a few 
Table 5. Priors to the pyaneti model of EPIC $212036875 \mathrm{~b}$ and results.

\begin{tabular}{|c|c|c|c|}
\hline Parameter & Units & Priors ${ }^{(a)}$ & Final value \\
\hline \multicolumn{4}{|c|}{ Fitted parameters } \\
\hline$T_{0}$ & Transit epoch (BJD $\left.{ }_{\mathrm{TDB}}-2450000\right)$ & $\mathcal{U}[8098.665,8098.695]$ & $8098.6791 \pm 0.0002$ \\
\hline$P_{\text {orb }}$ & Orbital period (days) & $\mathcal{U}[5.1679,5.1719]$ & $5.16992 \pm 0.00002$ \\
\hline$e$ & Eccentricity & $\mathcal{U}[0,0.3]$ & $0.134 \pm 0.002$ \\
\hline$\omega$ & Argument of periastron (degrees) & $\mathcal{U}[0,180]$ & $163 \pm 1$ \\
\hline$b$ & Impact parameter & $\mathcal{U}[0,1]$ & $0.920_{-0.006}^{+0.005}$ \\
\hline$a / R_{\star}$ & Scaled semi-major axis & $\mathcal{U}[1.1,15]$ & $9.2 \pm 0.2$ \\
\hline$R_{\mathrm{BD}} / R_{\star}$ & Scaled brown dwarf radius & $\mathcal{U}[0,0.1]$ & $0.0608 \pm 0.0009$ \\
\hline$K$ & Doppler semi-amplitude variation $\left(\mathrm{km} \mathrm{s}^{-1}\right)$ & $\mathcal{U}[0,15]$ & $5.289 \pm 0.013$ \\
\hline$q_{1}$ & Parameterised limb-darkening coefficient & $\mathcal{G}[0.38,0.10]$ & $0.41 \pm 0.10$ \\
\hline$q_{2}$ & Parameterised limb-darkening coefficient & $\mathcal{G}[0.26,0.10]$ & $0.26 \pm 0.10$ \\
\hline \multicolumn{4}{|c|}{ Derived parameters } \\
\hline$M_{\mathrm{BD}}$ & Brown dwarf mass $\left(M_{\mathrm{J}}\right)$ & $\cdots$ & $51 \pm 2$ \\
\hline$R_{\mathrm{BD}}$ & Brown dwarf radius $\left(R_{\mathrm{J}}\right)$ & $\ldots$ & $0.83 \pm 0.03$ \\
\hline$i^{(b)}$ & Inclination (degrees) & $\ldots$ & $83.9 \pm 0.2$ \\
\hline$a$ & Semi-major axis (AU) & $\ldots$ & $0.060 \pm 0.003$ \\
\hline$F$ & Insolation $\left(F_{\oplus}\right)$ & $\ldots$ & $740 \pm 50$ \\
\hline$\rho_{\star}{ }^{(c)}$ & Stellar density $\left(\mathrm{g} \mathrm{cm}^{-3}\right)$ & $\ldots$ & $0.55 \pm 0.04$ \\
\hline$\rho_{\mathrm{BD}}$ & Brown dwarf density $\left(\mathrm{g} \mathrm{cm}^{-3}\right)$ & $\ldots$ & $108_{-13}^{+15}$ \\
\hline $\log \left(g_{\mathrm{BD}}\right)$ & Brown dwarf surface gravity (cgs) & $\ldots$ & $5.23 \pm 0.02$ \\
\hline$T_{\text {eq }}(d)$ & Equilibrium temperature $(\mathrm{K})$ & $\cdots$ & $1450 \pm 30$ \\
\hline$T_{14}$ & Total transit duration $(\mathrm{h})$ & $\ldots$ & $2.17 \pm 0.01$ \\
\hline$T_{23}$ & Full transit duration $(\mathrm{h})$ & $\ldots$ & $0.76 \pm 0.09$ \\
\hline$u_{1}$ & Linear limb-darkening coefficient & $\cdots$ & $0.33 \pm 0.14$ \\
\hline$u_{2}$ & Quadratic limb-darkening coefficient & $\ldots$ & $0.30 \pm 0.13$ \\
\hline \multicolumn{4}{|c|}{ Additional parameters } \\
\hline$\gamma_{1}$ & Systemic velocity FIES1 $\left(\mathrm{km} \mathrm{s}^{-1}\right)$ & $\mathcal{U}[-27.1655,-16.5310]$ & $-21.26 \pm 0.03$ \\
\hline$\gamma_{2}$ & Systemic velocity FIES2 $\left(\mathrm{km} \mathrm{s}^{-1}\right)$ & $\mathcal{U}[-22.9664,-16.9289]$ & $-21.33 \pm 0.16$ \\
\hline$\gamma_{3}$ & Systemic velocity FIES3 $\left(\mathrm{km} \mathrm{s}^{-1}\right)$ & $\mathcal{U}[-27.3661,-16.5940]$ & $-21.30 \pm 0.01$ \\
\hline$\sigma_{\mathrm{F} 1}$ & RV jitter FIES1 $\left(\mathrm{km} \mathrm{s}^{-1}\right)$ & $\mathcal{U}[0,1]$ & $0.064_{-0.030}^{+0.046}$ \\
\hline$\sigma_{\mathrm{F} 2}$ & RV jitter FIES2 $\left(\mathrm{km} \mathrm{s}^{-1}\right)$ & $\mathcal{U}[0,1]$ & $0.202_{-0.129}^{+0.498}$ \\
\hline$\sigma_{\mathrm{F} 3}$ & RV jitter FIES3 $\left(\mathrm{km} \mathrm{s}^{-1}\right)$ & $\mathcal{U}[0,1]$ & $0.0092_{-0.006}^{+0.010}$ \\
\hline$\sigma_{\text {tr }}$ & Light curve jitter & $\mathcal{U}[0,0.00004733]$ & $0.000025 \pm 0.000005$ \\
\hline
\end{tabular}

Notes. ${ }^{(a)} \mathcal{U}[\mathrm{a}, \mathrm{b}]$ refers to uniform priors in the range $a-b$, and $\mathcal{G}[\mathrm{a}, \mathrm{b}]$ refers to Gaussian priors with mean $a$ and standard deviation $b .^{\left({ }^{b}\right)}$ Orbit inclination relative to the plane of the sky. ${ }^{(c)}$ Density from pyaneti transit modelling. Density from adopted stellar mass and radius is $0.58 \pm 0.08 \mathrm{~g} \mathrm{~cm}^{-3}$. ${ }^{(d)}$ Assuming isotropic re-radiation and a Bond albedo of zero. For example, by increasing the albedo to 0.3 and 0.6 , we find $T_{\mathrm{eq}} \approx 1310$ and $1140 \mathrm{~K}$, respectively.

tens of Jupiter masses (see reviews by Kratter \& Lodato 2016; Nayakshin 2017). We show in Appendix B and Fig. B.1 that gravitational instability can indeed give rise to fragments with the mass of EPIC 212036875 b. One of these fragments must then migrate to the present orbit of EPIC $212036875 \mathrm{~b}$, which can happen through Type I migration (Baruteau et al. 2011; Malik et al. 2015), although the extent of this is debated in the literature (Stamatellos 2015; Vorobyov \& Elbakyan 2018). On the other hand, gravitational instability often gives rise to more than one fragment, and in this case the dynamical interactions between fragments enhance their migration rate through the disc (Forgan et al. 2018). Indeed, the moderate eccentricity of EPIC 212036875 b may be a relic of these dynamical interactions, after some reduction by tidal forces.

\subsection{Tidal evolution of the system}

As the BD is on a close orbit with non-zero eccentricity, its orbit may be affected by tidal torques. These arise either from the deformation of the BD by the star (henceforth the BD tide) or from the deformation of the star by the BD (henceforth the stellar tide). These tides cause a change in both orbital 


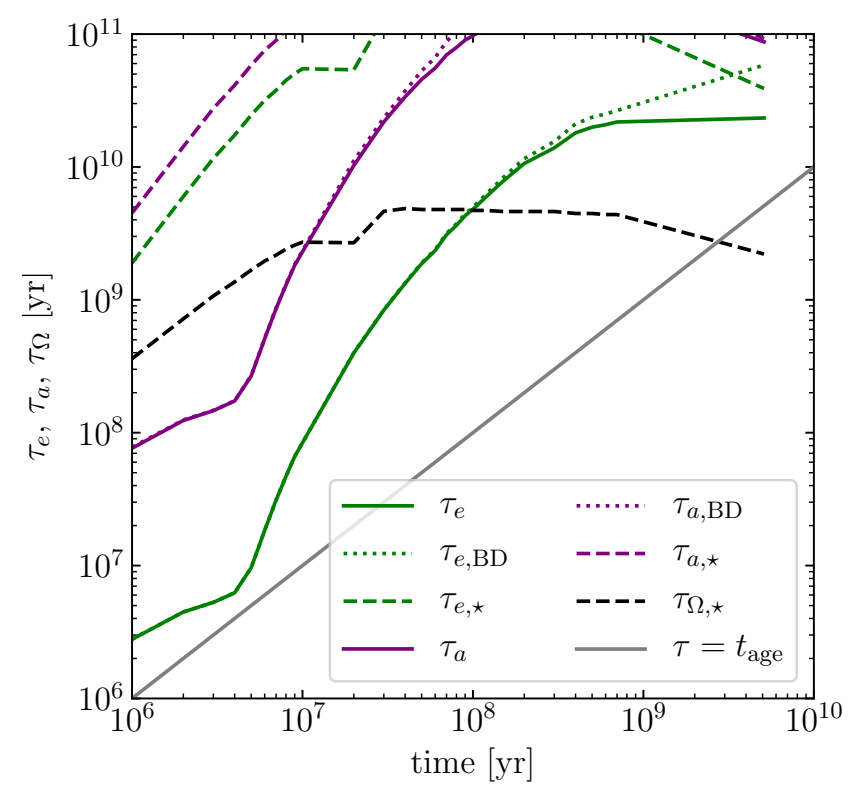

Fig. 7. Timescales for tidal orbital circularisation $\tau_{e}$, semi-major axis decay $\tau_{a}$, and stellar spin evolution, $\tau_{\Omega, \star}$. The contributions from the star and the brown dwarf as given by Eqs. (1)-(4) are shown, as well as the net effect, taking quality factors $Q_{\star}^{\prime}=10^{8}$ and $Q_{\mathrm{BD}}^{\prime}=10^{5}$. The diagonal line marks where the timescale at a given age is equal to the system's age.

semi-major axis and eccentricity, and hence there are four timescales to consider: the contributions of each tide to the decay of the semi-major axis and to the eccentricity. We use the tidal model of Jackson et al. (2008) and define the following timescales $\tau$ :

$$
\begin{aligned}
& \frac{1}{\tau_{a, \star}}=a_{\mathrm{BD}}^{-13 / 2} \frac{9}{2} \sqrt{\frac{G}{M_{\star}}} \frac{R_{\star}^{5} M_{\mathrm{BD}}}{Q_{\star}^{\prime}}, \\
& \frac{1}{\tau_{a, \mathrm{BD}}}=a_{\mathrm{BD}}^{-13 / 2} \frac{63}{2} \sqrt{G M_{\star}^{3}} \frac{R_{\mathrm{BD}}^{5} e_{\mathrm{BD}}^{2}}{Q_{\mathrm{BD}}^{\prime} M_{\mathrm{BD}}}, \\
& \frac{1}{\tau_{e, \star}}=a_{\mathrm{BD}}^{-13 / 2} \frac{171}{16} \sqrt{\frac{G}{M_{\star}}} \frac{R_{\star}^{5} M_{\mathrm{BD}}}{Q_{\star}^{\prime}}, \\
& \frac{1}{\tau_{e, \mathrm{BD}}}=a_{\mathrm{BD}}^{-13 / 2} \frac{63}{4} \sqrt{G M_{\star}^{3}} \frac{R_{\mathrm{BD}}^{5}}{Q_{\mathrm{BD}}^{\prime} M_{\mathrm{BD}}},
\end{aligned}
$$

where $Q_{\star}^{\prime}$ and $Q_{\mathrm{BD}}^{\prime}$ are the tidal quality factors of the star and the BD. We adopt quality factors of $10^{8}$ for the star, in line with the recent empirical calibration of Collier Cameron \& Jardine (2018) for stars in the equilibrium tide regime and with dynamical tide calculations for a $1.2 M_{\odot}$ F-type star by Ogilvie \& Lin (2007), and $10^{5}$ for the BD as inferred for Jupiter (Lainey et al. 2009). For simplicity, we hold $Q$ constant for both the star and the BD. In reality, $Q$ can exhibit a complicated dependence on the ratio of the periods of the orbit and of the stellar spin (see Fig. 8 of Barker \& Ogilvie 2009). We find that, with the current system parameters, the stellar tide dominates, and the decay timescales are $\tau_{a}=87 \mathrm{Gyr}$ and $\tau_{e}=23 \mathrm{Gyr}$. These values are longer than the system age, and hence the BD's orbit will not be currently tidally evolving. We note that the pre-print of Carmichael et al. (2019) gives a slightly longer circularisation time of 47 Gyr. The difference is largely due to their considering only the tide raised on the brown dwarf.
We note that the tidal timescales given in Eqs. (1)-(4) are extremely strong functions of the physical radii of the BD and of the star, so the tidal timescales change with system age (see, e.g., Zahn \& Bouchet 1989; Mathis 2015; Bolmont \& Mathis 2016). To explore the historical evolution of the tidal forces, we used the PHOENIX BT-Settl models (Baraffe et al. 2015) to obtain the radii of both the primary and the BD, and calculated the tidal timescales as a function of system age (see Fig. 7). This shows that for the system's main sequence lifetime the tidal forces have been negligible, but that the circularisation timescale was comparable to the system age at ages of a few Myr when the BD radius was several $R_{\mathrm{J}}$. Thus, it is possible that EPIC 212036875 b started tidally circularising early in its history and then stopped as its radius contracted.

A further issue relates to the evolution of the stellar spin: around $98 \%$ of the system's angular momentum lies in the BD's orbit, so it should spin the star up to (pseudo-)synchronisation ${ }^{8}$ if the timescale is short enough. For the present parameters, pseudo-synchronisation occurs at $\Omega_{\text {rot,ps }}=1.083 \pm 0.003 \Omega_{\text {orb }}$, far from the actual value of $\left(\Omega_{\text {rot,actual }} / \Omega_{\text {orb }}=0.69\right)$. With $Q_{\star}^{\prime}=10^{8}$ we find a timescale for spin evolution of $\tau_{\Omega, \star}=2.2 \mathrm{Gyr}$, comparable to the system age. Given that the star is not pseudosynchronised, this implies that $Q_{\star}^{\prime} \gtrsim 10^{8}$. In principle, $Q_{\star}^{\prime}$ can be determined by transit timing variations, but this is challenging: from Eq. (7) in Birkby et al. (2014), we estimate that transits would occur just $1 \mathrm{~s}$ earlier after $20 \mathrm{yr}$ even if $Q_{\star}^{\prime}=10^{7}$. Alternatively, magnetic effects such as magnetic breaking may force the system away from pseudo-synchronisation: magnetic fields are possessed by both BDs (of kG or stronger: Kao et al. 2018; Berdyugina et al. 2017; Metodieva et al. 2017) and F stars (e.g. Mathur et al. 2014; Augustson et al. 2013). The stellar wind and the magnetism of the BD, studied in Ferraz-Mello et al. (2015), among others, can also interplay, as can induction heating (Kislyakova et al. 2018).

We summarise a potential formation and evolution history for this system: EPIC 212036875 b formed through gravitational instability early in the protoplanetary disc's evolution. It may have formed as one of several similar objects, the others either ejected by dynamical interactions or undetectable given current data. The interactions with the other objects would have excited the orbital eccentricity of EPIC 212036875 b, and hastened its migration towards the primary star in the few Myr of the protoplanetary disc's lifetime. At this young age, the BD's large radius may have led to some tidal decay of its orbital eccentricity, but after several Myr its radius would have shrunk sufficiently to weaken tidal forces enough to freeze its orbit in place. Finally, the tide raised on the star by the BD may have begun forcing the star towards spin-orbit pseudo-synchronisation during the star's main sequence lifetime, but this process has not yet finished.

\subsection{Mass-density diagram}

In order to investigate possible differences between BDs and GPs, we show a mass-density diagram in Fig. 8 for planets ${ }^{9}$ and $\mathrm{BDs}^{10}$. It should be noted that all these objects have close-in orbits to the host star (most have $P_{\text {orb }}<10$ days). Also shown are eclipsing low-mass stars (Ribas 2003; Bouchy et al. 2005;

8 Pseudo-synchronisation occurs for eccentric orbits where the spin angular velocity locks to a value given by Eq. (42) of Hut (1981). The exact value is a function of eccentricity and orbital frequency.

9 Well-studied planets listed at http://www.astro.keele.ac.uk/ jkt/tepcat/.

${ }^{10}$ Discovered by space- and ground-based transit searches (see references in Sect. 1). 


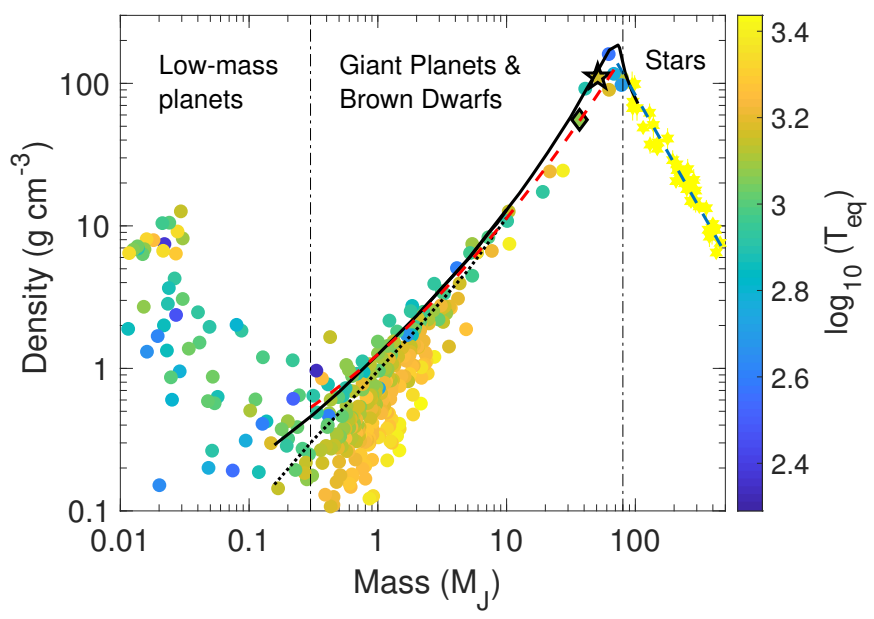

Fig. 8. Mass-density diagram for planets, brown dwarfs, and low-mass stars in eclipsing binaries with a precision in measured mass and density better than $20 \%$. The star and diamond symbols mark the locations of EPIC $212036875 \mathrm{~b}$ and EPIC $219388192 \mathrm{~b}$ also found by our programme (Nowak et al. 2017). The red dashed line represents a second-order polynomial fit to the data with $M=0.3-80 M_{\mathrm{J}}$ and equilibrium temperatures $<1000 \mathrm{~K}$. The blue dashed line shows a linear fit to the stars with $M>80 M_{\mathrm{J}}$. The nominal separation at $80 M_{\mathrm{J}}$ between brown dwarfs and stars, and the empirical separation between low-mass and giant planets at $0.3 M_{\mathrm{J}}$ are marked with the vertical dash-dotted lines. The solid black line shows the theoretical relationship for $\mathrm{H} / \mathrm{He}$ dominated giant objects with $Z=0.02$, age $=5 \mathrm{Gyr}$, without irradiation (Baraffe et al. 2003, 2008), and the dotted black line the same model including irradiation from a solar-type star at 0.045 AU (Baraffe et al. 2008).

Pont et al. 2005, 2006; Demory et al. 2009; Tal-Or et al. 2013; Zhou et al. 2014; Díaz et al. 2014; Chaturvedi et al. 2016 and references in Table 1; Gillen et al. 2017; von Boetticher et al. 2017; Shporer et al. 2017; Chaturvedi et al. 2018 and references in Table 4; Carmichael et al. 2019) up to $450 M_{\mathrm{J}}\left(0.43 M_{\odot}\right)$ mostly from ground-based discoveries. We only include objects with a precision in mass and density better than $20 \%$ (in total 253 GPs and BDs, and 43 low-mass stars). The vertical dash-dotted line at $80 M_{\mathrm{J}}$ indicates the nominal separation between BDs and nuclear burning $\mathrm{M}$ dwarfs. The colours of the planets and BDs indicate the logarithm of the equilibrium temperatures, $T_{\text {eq }}$. It can be clearly seen that low-mass GPs with high incident flux, and thus high $T_{\text {eq }}$, have lower densities, which could be a sign of inflated radii due to the proximity to the host stars (Miller \& Fortney 2011; Baraffe et al. 2014; Tremblin et al. 2017). We fitted a second-order polynomial to the data (red dashed line) between $0.3 M_{\mathrm{J}}$ and $80 M_{\mathrm{J}}$ for objects with $T_{\text {eq }}<1000 \mathrm{~K}$ to exclude objects with inflated radii (Weiss et al. 2013), in total 33 objects, and found $\log \rho=0.16 \times \log ^{2}(M)+0.80 \times \log (M)+0.10$. The blue dashed line shows a linear fit to the stars with $M>80 M_{\mathrm{J}}$ : $\log (\rho)=-1.6 \times \log (M)+5.1$. Compared to Hatzes \& Rauer (2015), we now find a sharp turnover at $\sim 73 M_{\mathrm{J}}$ instead of $\sim 60 M_{\mathrm{J}}$. The empirical fit follows closely the theoretical relationship for $\mathrm{H} / \mathrm{He}$ dominated GPs (Baraffe et al. 2008) and BDs (Baraffe et al. 2003) with $Z=0.02$, age $=5$ Gyr and without irradiation drawn with a solid black line. The dotted black line shows the same model for GPs including irradiation from a solar-type star at $a=0.045$ AU (Baraffe et al. 2008), which clearly shows the impact of irradiation for the lower mass GPs. At the lower end, we find a turnover at $\sim 0.3 M_{\mathrm{J}}$ in agreement with Hatzes \& Rauer (2015), marking the transition to low-mass planets. Our results are in agreement with Chen \& Kipping (2017) who found $R \sim M^{-0.04}$ for objects between $0.4 M_{\mathrm{J}}$ and $80 M_{\mathrm{J}}$.
Our two BDs fall close to the theoretical model for $\mathrm{H} / \mathrm{He}$ dominated BDs and to the empirical fit. No distinguishing features between GPs and BDs can be seen. After a brief phase lasting $\sim 10 \mathrm{Myr}$ when the deuterium and lithium fusion halts contraction, BDs cool and contract in a way similar to GPs. This suggests that both types of objects will follow the same trend in the mass-density diagram independent of the formation mechanism, especially at late ages. At earlier stages, the difference in radius is larger (e.g. Baraffe et al. 2008) and contributes to the scatter of the data points.

\section{Conclusions}

We report the discovery and characterisation of a rare object with a mass of $51 \pm 2 M_{\mathrm{J}}$ and a radius of $0.83 \pm 0.03 R_{\mathrm{J}}$ in an eccentric 5.17-day orbit around the slightly evolved F7 V star EPIC 212036875. Since the star is seen close to equator-on, future observations with large 8-10 m class telescopes, could allow the measurement of the (3D) obliquity angle between the stellar rotation axis and the brown dwarf orbit axis via the Rossiter-McLaughlin effect. Thanks to the evolutionary state of the host star, this is one of the few transiting brown dwarfs for which a relatively precise age can be estimated. Our results are in agreement with Carmichael et al. (2019) who recently reported an independent discovery and characterisation of EPIC 212036875 b.

We show with a simple analytical model that formation of a brown dwarf of the required mass is possible at several tens of AU through gravitational instability, although significant orbital migration is required to bring the object to its current orbit. The orbit may have experienced a period of tidal circularisation within the first few Myr of the system's life when the brown dwarf's radius was very much larger than it is at present, which ceased as its physical radius contracted. The stellar spin may have been affected by the tidal torque from the brown dwarf during the system's main sequence lifetime, but the lack of spin-orbit synchronisation points to a weak stellar dissipation parameter $\left(Q_{\star}^{\prime} \gtrsim 10^{8}\right)$. There is also a possibility that magnetic field plays a role here, which could change this estimate.

We find no distinction between brown dwarfs and giant planets based on the mass-density diagram. This supports the previous suggestion by Hatzes \& Rauer (2015), and supported by Chen \& Kipping (2017), that brown dwarfs could simply represent the high-mass end of giant planets and that there are no observable differences between mature brown dwarfs and giant planets. The brown-dwarf desert may be a reflection of the decreasing number of objects towards the high-mass end of the giant planet distribution formed by core-accretion, and the low-mass end of stars formed by gravitational instabilities.

Acknowledgements. This paper is based on observations obtained with (a) the MuSCAT2 instrument, developed by ABC, at Telescopio Carlos Sánchez operated on the island of Tenerife by the IAC in the Spanish Observatorio del Teide; (b) the Nordic Optical Telescope (NOT) operated on the island of La Palma jointly by Denmark, Finland, Iceland, Norway, and Sweden, in the Spanish Observatorio del Roque de los Muchachos (ORM) of the Instituto de Astrodísica de Canarias (IAC, CAT, and TAC programmes 57-015, 57-206, and 57-210; OPTICON programmes 2018A-044 and 2018B-052; and the Spanish-Nordic programme 58-301); (c) the McDonald observatory operated by The University of Texas at Austin; (d) the Subaru Telescope operated by the National Astronomical Observatory of Japan; (e) NESSI, funded by the NASA Exoplanet Exploration Program and the NASA Ames Research Center. NESSI was built at the Ames Research Center by Steve B. Howell, Nic Scott, Elliott P. Horch, and Emmett Quigley. This paper includes data collected by the K2 mission. Funding for the K2 mission is provided by the NASA Science Mission directorate. We thank the NOT, McDonald, Subaru, and NESSI staff members for their support during the observations. This work has made use of the SME package, which 
benefits from the continuing development work by J. Valenti and N. Piskunov, and we gratefully acknowledge their continued support. (Kupka et al. 2000; Ryabchikova et al. 2015). C.M.P., M.F. and I.G. gratefully acknowledge the sup port of the Swedish National Space Agency (DNR 163/16 and 174/18). Sz.Cs thanks the Hungarian National Research, Development and Innovation Office for the NKFI-KH-130372 grants. A.J.M. and M.B.D. acknowledge support from the IMPACT grant from the Knut and Alice Wallenberg Foundation (2014.0017). J.K., S.G., M.P., S.C., A.P.H., K.W.F.L., M.E., and H.R. acknowledge support by DFG grants PA525/18-1, PA525/19-1, PA525/20-1, HA 3279/12-1, and RA 714/14-1 within the DFG Schwerpunkt SPP 1992, "Exploring the Diversity of Extrasolar Planets". G.N., H.J.D., and D.N. acknowledge support from grants ESP2015-65712-C5-4-R and ESP2017-87676-C5-4-R from the Spanish Secretary of State for R\&D\&i (MINECO). P.G.B. acknowledges support from the MINECO-postdoctoral fellowship programme "Juan de la Cierva Incorporacion" (IJCI-2015-26034). P.K. acknowledges support from GACR 17-01752J. M.S acknowledges the Postdoc@MUNI project CZ.02.2.69/0.0/0.0/16-027/0008360. I.R. acknowledges support from the Spanish Ministry for Science, Innovation, and Universities (MCIU) and the Fondo Europeo de Desarrollo Regional (FEDER) through grant ESP2016-80435-C2-1-R, as well as the support of the Generalitat de Catalunya/CERCA programme. N.N. acknowledges support from JSPS KAKENHI Grant Numbers JP18H01265 and 18H05439, and JST PRESTO Grant Number JPMJPR1775. L.G.C. acknowledges support from the MINECO FPI-SO doctoral research project SEV-2015-0548-17-2 and predoctoral contract BES-2017-082610. We thank the anonymous referee whose constructive comments led to an improvement of the paper.

\section{References}

Alibert, Y., Mordasini, C., \& Benz, W. 2004, A\&A, 417, L25

Augustson, K. C., Brun, A. S., \& Toomre, J. 2013, ApJ, 777, 153

Baraffe, I., Chabrier, G., Allard, F., \& Hauschildt, P. H. 2002, A\&A, 382, 563

Baraffe, I., Chabrier, G., Barman, T. S., Allard, F., \& Hauschildt, P. H. 2003, A\&A, 402, 701

Baraffe, I., Chabrier, G., \& Barman, T. 2008, A\&A, 482, 315

Baraffe, I., Chabrier, G., Fortney, J., \& Sotin, C. 2014, Protostars and Planets VI (Tucson, AZ: University of Arizona Press), 763

Baraffe, I., Homeier, D., Allard, F., \& Chabrier, G. 2015, A\&A, 577, A42

Barker, A. J., \& Ogilvie, G. I. 2009, MNRAS, 395, 2268

Barragán, O., \& Gandolfi, D. 2017, Astrophysics Source Code Library [record ascl:1706.001]

Barragán, O., Gandolfi, D., Smith, A. M. S., et al. 2018a, MNRAS, 475, 1765

Barragán, O., Gandolfi, D., Dai, F., et al. 2018b, A\&A, 612, A95

Barragán, O., Gandolfi, D., \& Antoniciello, G. 2019, MNRAS, 482, 1017

Baruteau, C., Meru, F., \& Paardekooper, S.-J. 2011, MNRAS, 416, 1971

Bayliss, D., Hojjatpanah, S., Santerne, A., et al. 2017, AJ, 153, 15

Berdyugina, S. V., Harrington, D. M., Kuzmychov, O., et al. 2017, ApJ, 847, 61

Birkby, J. L., Cappetta, M., Cruz, P., et al. 2014, MNRAS, 440, 1470

Bolmont, E., \& Mathis, S. 2016, Celest. Mech. Dyn. Astron., 126, 275

Borucki, W. J. 2018, Handbook of Exoplanets (Cham: Springer International Publishing), 80

Bouchy, F., Pont, F., Melo, C., et al. 2005, A\&A, 431, 1105

Brandeker, A., \& Cataldi, G. 2019, A\&A, 621, A86

Bressan, A., Marigo, P., Girardi, L., et al. 2012, MNRAS, 427, 127

Bruntt, H., Bedding, T. R., Quirion, P.-O., et al. 2010, MNRAS, 405, 1907

Buchhave, L. A., Bakos, G. Á., Hartman, J. D., et al. 2010, ApJ, 720, 1118

Burrows, A., Hubbard, W. B., Lunine, J. I., \& Liebert, J. 2001, Rev. Mod. Phys., 73, 719

Cañas, C. I., Bender, C. F., Mahadevan, S., et al. 2018, ApJ, 861, L4

Carmichael, T., Latham, D., \& Vanderburg, A. 2019, AJ, 158, 38

Chabrier, G., Johansen, A., Janson, M., \& Rafikov, R. 2014, Protostars and Planets VI (Tucson, AZ: University of Arizona Press), 619

Chaturvedi, P., Chakraborty, A., Anandarao, B. G., Roy, A., \& Mahadevan, S. 2016, MNRAS, 462, 554

Chaturvedi, P., Sharma, R., Chakraborty, A., Anandarao, B. G., \& Prasad, N. J. S. S. V. 2018, AJ, 156, 27

Chen, J., \& Kipping, D. 2017, ApJ, 834, 17

Claret, A., \& Bloemen, S. 2011, A\&A, 529, A75

Collier Cameron, A., \& Jardine, M. 2018, MNRAS, 476, 2542

Cox, A. N. 2000, Allen's Astrophysical Quantities (New York: Springer)

Csizmadia, S. 2016, III.6 Exploration of the Brown Dwarf Regime Around Solarlike Stars by CoRoT, ed. CoRoT Team (France: EDP Sciences), 143

Csizmadia, S., Pasternacki, T., Dreyer, C., et al. 2013, A\&A, 549, A9

da Silva, L., Girardi, L., Pasquini, L., et al. 2006, A\&A, 458, 609

Deleuil, M., \& Fridlund, M. 2018, Handbook of Exoplanets (Cham: Springer International Publishing), 79

Deleuil, M., Deeg, H. J., Alonso, R., et al. 2008, A\&A, 491, 889

Demory, B.-O., Ségransan, D., Forveille, T., et al. 2009, A\&A, 505, 205
Díaz, R. F., Montagnier, G., Leconte, J., et al. 2014, A\&A, 572, A109

Dieterich, S. B., Henry, T. J., Jao, W.-C., et al. 2014, AJ, 147, 94

Doyle, A. P., Davies, G. R., Smalley, B., Chaplin, W. J., \& Elsworth, Y. 2014, MNRAS, 444, 3592

Eastman, J., Gaudi, B. S., \& Agol, E. 2013, PASP, 125, 83

Endl, M., \& Cochran, W. D. 2016, PASP, 128, 094502

Ferraz-Mello, S., Tadeu dos Santos, M., Folonier, H., et al. 2015, ApJ, 807, 78

Fletcher, M., Nayakshin, S., Stamatellos, D., et al. 2019, MNRAS, 486, 4398

Forgan, D. H., Hall, C., Meru, F., \& Rice, W. K. M. 2018, MNRAS, 474, 5036

Frandsen, S., \& Lindberg, B. 1999, Astrophysics with the NOT, eds.

H. Karttunen \& V. Piirola (Piikkio: University of Turku), 71

Fridlund, M. 2018, Space Missions for Exoplanet Research: Overview and Introduction (Cham: Springer), 77

Fridlund, M., Gaidos, E., Barragán, O., et al. 2017, A\&A, 604, A16

Gandolfi, D., Parviainen, H., Deeg, H. J., et al. 2015, A\&A, 576, A11

Gandolfi, D., Barragán, O., Livingston, J. H., et al. 2018, A\&A, 619, L10

Gandolfi, D., Fossati, L., Livingston, J. H., et al. 2019, ApJ, 876, L24

Gillen, E., Hillenbrand, L. A., David, T. J., et al. 2017, ApJ, 849, 11

Gramajo, L. V., Rodón, J. A., \& Gómez, M. 2014, AJ, 147, 140

Grether, D., \& Lineweaver, C. H. 2006, ApJ, 640, 1051

Grziwa, S., Pätzold, M., \& Carone, L. 2012, MNRAS, 420, 1045

Hatzes, A. P., \& Rauer, H. 2015, ApJ, 810, L25

Hennebelle, P., \& Chabrier, G. 2008, ApJ, 684, 395

Hirano, T., Fukui, A., Mann, A. W., et al. 2016, ApJ, 820, 41

Hirano, T., Dai, F., Gandolfi, D., et al. 2018, AJ, 155, 127

Hjorth, M., Justesen, A. B., Hirano, T., et al. 2019, MNRAS, 484, 3522

Hodžić, V., Triaud, A. H. M. J., Anderson, D. R., et al. 2018, MNRAS, 481 5091

Howell, S. B., Everett, M. E., Sherry, W., Horch, E., \& Ciardi, D. R. 2011, AJ, 142,19

Huber, D., Bryson, S. T., Haas, M. R., et al. 2016, ApJS, 224, 2

Hut, P. 1981, A\&A, 99, 126

Ida, S., Guillot, T., \& Morbidelli, A. 2016, A\&A, 591, A72

Irwin, J. M., Charbonneau, D., Esquerdo, G. A., et al. 2018, AJ, 156, 140

Jackson, B., Greenberg, R., \& Barnes, R. 2008, ApJ, 678, 1396

Johnston, W. R. 2015, http://www.johnstonsarchive.net/astro/ browndwarflist.html

Kao, M. M., Hallinan, G., Pineda, J. S., Stevenson, D., \& Burgasser, A. 2018, ApJS, 237, 25

Kipping, D. M. 2010, MNRAS, 408, 1758

Kipping, D. M. 2013, MNRAS, 435, 2152

Kislyakova, K. G., Fossati, L., Johnstone, C. P., et al. 2018, ApJ, 858, 105

Kobayashi, N., Tokunaga, A. T., Terada, H., et al. 2000, Proc. SPIE, 4008, 1056

Korth, J., Csizmadia, S., Gandolfi, D., et al. 2019, MNRAS, 482, 1807

Kovács, G., Zucker, S., \& Mazeh, T. 2002, A\&A, 391, 369

Kratter, K., \& Lodato, G. 2016, ARA\&A, 54, 271

Kuerster, M., Schmitt, J. H. M. M., Cutispoto, G., \& Dennerl, K. 1997, A\&A, 320,831

Kupka, F. G., Ryabchikova, T. A., Piskunov, N. E., Stempels, H. C., \& Weiss, W. W. 2000, Balt. Astron., 9, 590

Kurucz, R. L. 2013, Astrophysics Source Code Library [record ascl:1303.024]

Lainey, V., Arlot, J.-E., Karatekin, Ö., \& van Hoolst T. 2009, Nature, 459, 957

Lambrechts, M., \& Johansen, A. 2012, A\&A, 544, A32

Lang, D., Hogg, D. W., Mierle, K., Blanton, M., \& Roweis, S. 2010, AJ, 139, 1782

Livingston, J. H., Dai, F., Hirano, T., et al. 2019, MNRAS, 484, 8

Ma, B., \& Ge, J. 2014, MNRAS, 439, 2781

Malik, M., Meru, F., Mayer, L., \& Meyer, M. 2015, ApJ, 802, 56

Mandel, K., \& Agol, E. 2002, ApJ, 580, L171

Marcy, G. W., \& Butler, R. P. 2000, PASP, 112, 137

Mathis, S. 2015, A\&A, 580, L3

Mathur, S., García, R. A., Ballot, J., et al. 2014, A\&A, 562, A124

McQuillan, A., Mazeh, T., \& Aigrain, S. 2014, ApJS, 211, 24

Metodieva, Y. T., Kuznetsov, A. A., Antonova, A. E., et al. 2017, MNRAS, 465, 1995

Miller, N., \& Fortney, J. J. 2011, ApJ, 736, L29

Mordasini, C., Alibert, Y., Georgy, C., et al. 2012, A\&A, 547, A112

Narita, N., Fukui, A., Kusakabe, N., et al. 2019, J. Astron. Telesc. Instrum. Syst., 5, 015001

Nayakshin, S. 2017, PASA, 34, e002

Nowak, G., Palle, E., Gandolfi, D., et al. 2017, AJ, 153, 131

Ofir, A. 2014, A\&A, 561, A138

Ogilvie, G. I., \& Lin, D. N. C. 2007, ApJ, 661, 1180

Padoan, P., \& Nordlund, A. 2004, ApJ, 617, 559

Palle, E., Nowak, G., Luque, R., et al. 2019, A\&A, 623, A41

Parviainen, H. 2015, MNRAS, 450, 3233

Parviainen, H., \& Aigrain, S. 2015, MNRAS, 453, 3822

Persson, C. M., Fridlund, M., Barragán, O., et al. 2018, A\&A, 618, A33 
Piskunov, N., \& Valenti, J. A. 2017, A\&A, 597, A16

Pollack, J. B., Hubickyj, O., Bodenheimer, P., et al. 1996, Icarus, 124, 62

Pont, F., Bouchy, F., Melo, C., et al. 2005, A\&A, 438, 1123

Pont, F., Moutou, C., Bouchy, F., et al. 2006, A\&A, 447, 1035

Ribas, I. 2003, A\&A, 398, 239

Rice, W. K. M., \& Armitage, P. J. 2003, ApJ, 598, L55

Robitaille, T. P., Whitney, B. A., Indebetouw, R., \& Wood, K. 2007, ApJS, 169 , 328

Ryabchikova, T., Piskunov, N., Kurucz, R. L., et al. 2015, Phys. Scr., 90, 054005

Sahlmann, J., Ségransan, D., Queloz, D., et al. 2011, A\&A, 525, A95

Scott, N. J., \& Howell, S. B. 2018, SPIE, 10701, 107010G

Shporer, A., Zhou, G., Vanderburg, A., et al. 2017, ApJ, 847, L18

Siverd, R. J., Beatty, T. G., Pepper, J., et al. 2012, ApJ, 761, 123

Skrzypek, N., Warren, S. J., \& Faherty, J. K. 2016, A\&A, 589, A49

Southworth, J. 2011, MNRAS, 417, 2166

Spiegel, D. S., Burrows, A., \& Milsom, J. A. 2011, ApJ, 727, 57

Stamatellos, D. 2015, ApJ, 810, L11

Tal-Or, L., Mazeh, T., Alonso, R., et al. 2013, A\&A, 553, A30

Telting, J. H., Avila, G., Buchhave, L., et al. 2014, Astron. Nachr., 335, 41

Toomre, A. 1964, ApJ, 139, 1217

Torres, G., Andersen, J., \& Giménez, A. 2010, A\&ARv, 18, 67

Tremblin, P., Chabrier, G., Mayne, N. J., et al. 2017, ApJ, 841, 30

Triaud, A. H. M. J. 2018, Handbook of Exoplanets (Cham: Springer), 2

Triaud, A. H. M. J., Queloz, D., Bouchy, F., et al. 2009, A\&A, 506, 377

Triaud, A. H. M. J., Hebb, L., Anderson, D. R., et al. 2013, A\&A, 549, A18

Triaud, A. H. M. J., Martin, D. V., Ségransan, D., et al. 2017, A\&A, 608, A129

Udry, S., Mayor, M., \& Queloz, D. 1999, Precise Stellar Radial Velocities: IAU Colloquium. 170, eds. J. B. Hearnshaw \& C. D. Scarfe, in ASP Conf. Ser, 185,367

Valenti, J. A., \& Piskunov, N. 1996, A\&AS, 118, 595

Vanderburg, A., \& Johnson, J. A. 2014, PASP, 126, 948

von Boetticher, A., Triaud, A. H. M. J., Queloz, D., et al. 2017, A\&A, 604, L6

Vorobyov, E. I., \& Elbakyan, V. G. 2018, A\&A, 618, A7

Weiss, L. M., Marcy, G. W., Rowe, J. F., et al. 2013, ApJ, 768, 14

Whitworth, A. P. 2018, Brown Dwarf Formation: Theory (Cham: Springer), 95

Winn, J. N. 2010, Exoplanet Transits and Occultations, ed. S. Seager (Tucson, AZ: University of Arizona Press), 55

Winn, J. N., Holman, M. J., Henry, G. W., et al. 2007, AJ, 133, 1828

Zahn, J. P., \& Bouchet, L. 1989, A\&A, 223, 112

Zechmeister, M., \& Kürster, M. 2009, A\&A, 496, 577

Zhou, G., Bayliss, D., Hartman, J. D., et al. 2014, MNRAS, 437, 283

Zhou, G., Bakos, G. Á., Bayliss, D., et al. 2019, AJ, 157, 31

1 Department of Space, Earth and Environment, Onsala Space Observatory, Chalmers University of Technology, SE-439 92 Onsala, Sweden.

e-mail: carina. persson@chalmers. se

2 Institute of Planetary Research, German Aerospace Center (DLR), Rutherfordstrasse 2, 12489 Berlin, Germany

3 Lund Observatory, Department of Astronomy \& Theoretical Physics, Lund University, Box 43, SE-221 00 Lund, Sweden

4 Leiden Observatory, University of Leiden, PO Box 9513, 2300 RA, Leiden, The Netherlands
5 Thüringer Landessternwarte Tautenburg, 07778 Tautenburg, Germany

${ }^{6}$ Instituto de Astrofísica de Canarias (IAC), 38205 La Laguna, Tenerife, Spain

7 Departamento de Astrofísica, Universidad de La Laguna, 38206 La Laguna, Tenerife, Spain

${ }^{8}$ Dipartimento di Fisica, Università di Torino, Via Pietro Giuria 1, 10125 Torino, Italy

9 Department of Astronomy, The University of Tokyo, 7-3-1 Hongo, Bunkyo-ku, Tokyo 113-0033, Japan

${ }^{10}$ Department of Astronomy and McDonald Observatory, University of Texas at Austin, 2515 Speedway, Austin, TX 78712, USA

11 Department of Earth and Planetary Sciences, Tokyo Institute of Technology, 2-12-1 Ookayama, Meguro-ku, Tokio, Japan

${ }^{12}$ Rheinisches Institut für Umweltforschung an der Universität $\mathrm{zu}$ Köln, Aachener Strasse 209, 50931 Köln, Germany

13 Stellar Astrophysics Centre, Department of Physics and Astronomy, Aarhus University, Ny Munkegade 120, 8000 Aarhus C, Denmark

${ }^{14}$ Department of Astronomy, The Ohio State University, 140 West 18th Ave., Columbus, OH 43210, USA

15 Sub-department of Astrophysics, Department of Physics, University of Oxford, Oxford OX1 3RH, UK

16 Department of Astrophysical Sciences, Princeton University, 4 Ivy Lane, Princeton, NJ 08544, USA

17 Institute für Physik, Geophysik, Astrophysik und Meteorologie, Karl-Franzens Universität Graz, Université-Platz 5, 8010 Graz, Austria

18 Astronomy Department and Van Vleck Observatory, Wesleyan University, Middletown, CT 06459, USA

${ }^{19}$ Department of Physics and Kavli Institute for Astrophysics and Space Research, MIT, Cambridge, MA 02139, USA

${ }^{20}$ National Astronomical Observatory of Japan, NINS, 2-21-1 Osawa, Mitaka, Tokyo 181-8588, Japan

${ }^{21}$ Astronomical Institute, Czech Academy of Sciences, Fričova 298, 25165, Ondřejov, Czech Republic

22 Astrobiology Center, NINS, 2-21-1 Osawa, Mitaka, Tokyo 1818588, Japan

${ }^{23}$ Center for Astronomy and Astrophysics, TU Berlin, Hardenbergstr. 36, 10623 Berlin, Germany

24 JST, PRESTO, 7-3-1 Hongo, Bunkyo-ku, Tokyo 113-0033, Japan

25 Department of Earth, Atmospheric and Planetary Sciences, MIT, 77 Massachusetts Avenue, Cambridge, MA 02139, USA

${ }^{26}$ Institut de Ciències de l'Espai (ICE, CSIC), Campus UAB,C/ de Can Magrans s/n, 08193 Bellaterra, Spain

27 Institut d'Estudis Espacials de Catalunya (IEEC), C/ Gran Capità 2-4, 08034 Barcelona, Spain

28 Department of Theoretical Physics and Astrophysics, Masaryk University, Kotlářská 2, 61137 Brno, Czech Republic

29 Astronomical Institute, Faculty of Mathematics and Physics, Charles University, Ke Karlovu 2027/3, 12116 Prague, Czech Republic 
C. M. Persson et al.: Greening of the brown-dwarf desert

\section{Appendix A: Additional table and figures}

Table A.1. FIES RV measurements of EPIC 212036875.

\begin{tabular}{|c|c|c|}
\hline \multirow{2}{*}{$\begin{array}{l}\mathrm{BJD}_{\mathrm{TDB}}^{(a)} \\
(-2450000.0) \\
\end{array}$} & \multicolumn{2}{|c|}{ RV } \\
\hline & $\left(\mathrm{km} \mathrm{s}^{-1}\right)$ & $\left(\mathrm{km} \mathrm{s}^{-1}\right)$ \\
\hline FIES 1 & Value & Err \\
\hline 8218.479167 & -27.0655 & 0.0490 \\
\hline 8220.404275 & -18.5041 & 0.0458 \\
\hline 8221.487928 & -16.6310 & \\
\hline 892 & -19 & 0.0523 \\
\hline 8233.440969 & -24.5166 & 0.0364 \\
\hline 185 & 38 & 0.0465 \\
\hline \multicolumn{3}{|l|}{ FIES 2} \\
\hline 230 & 64 & 0.0877 \\
\hline & -17 & 0.0563 \\
\hline 8247.446950 & -17.0289 & 0.0730 \\
\hline \multicolumn{3}{|l|}{ FIES 3} \\
\hline 825140 & 1 & .0357 \\
\hline & -16.6940 & 0.0259 \\
\hline 8253.439344 & -19.7521 & 0.0260 \\
\hline 8257.445193 & -16.6979 & 0.0259 \\
\hline 825 & -18 & 0.0202 \\
\hline 8260. & -26 & 0.0294 \\
\hline 8261.435608 & -20.1255 & 0.0259 \\
\hline 8518.672339 & -26.9764 & 0.0401 \\
\hline 8522.638533 & -22.1673 & 0.0255 \\
\hline 8523.66278 & -27.2661 & 0.0228 \\
\hline 8524.698470 & -22.4489 & 0.0242 \\
\hline 8539.620870 & -25.9885 & 0.0264 \\
\hline 8540.645300 & -19.9479 & 0.0337 \\
\hline 8541.620968 & -16.8566 & 0.0251 \\
\hline
\end{tabular}

Notes. ${ }^{(a)}$ Barycentric Julian day in barycentric dynamical time.

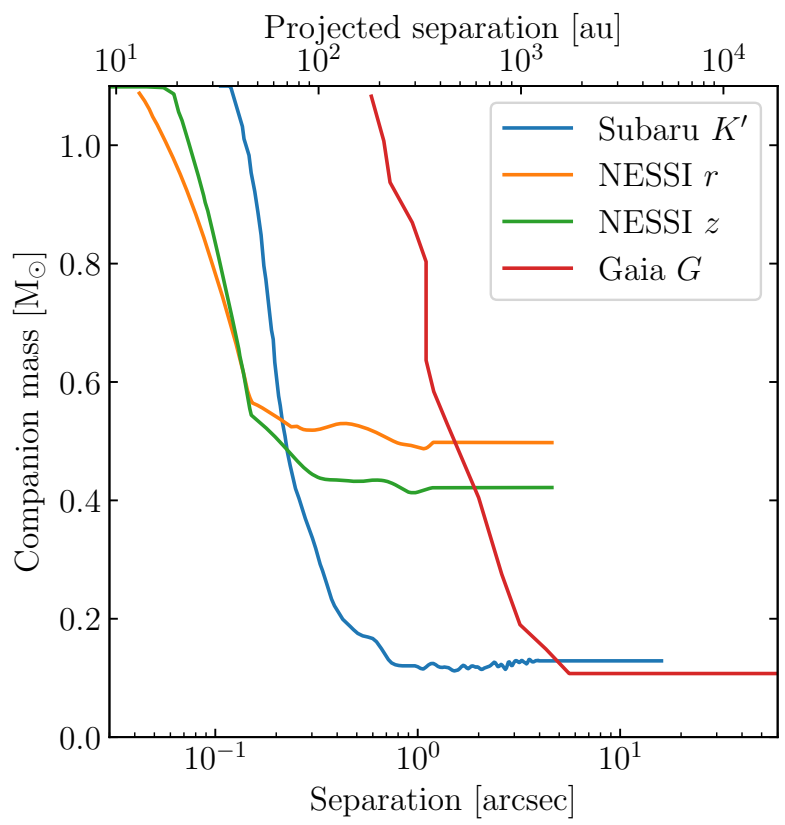

Fig. A.1. Limits of companion masses as a function of separation in arcsec and projected separation computed with the Baraffe et al. (2015) models for our NESSI and Subaru imaging, and the Gaia 50\% detectability limit from Brandeker \& Cataldi (2019).

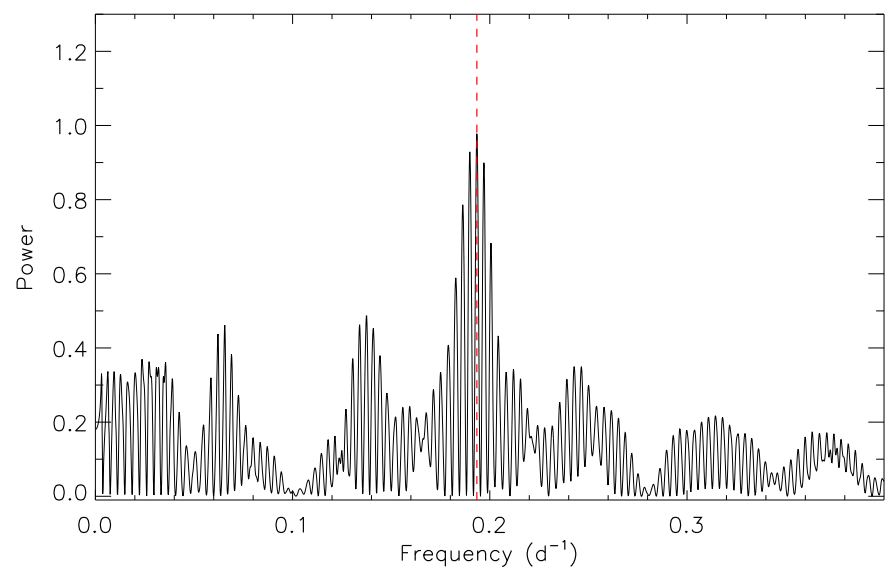

Fig. A.2. Generalised Lomb-Scargle periodogram of the combined FIES RVs. The red dashed line indicates the orbital frequency of the brown dwarf. Shown are the one-year aliases symmetrically distributed around the orbital frequency.
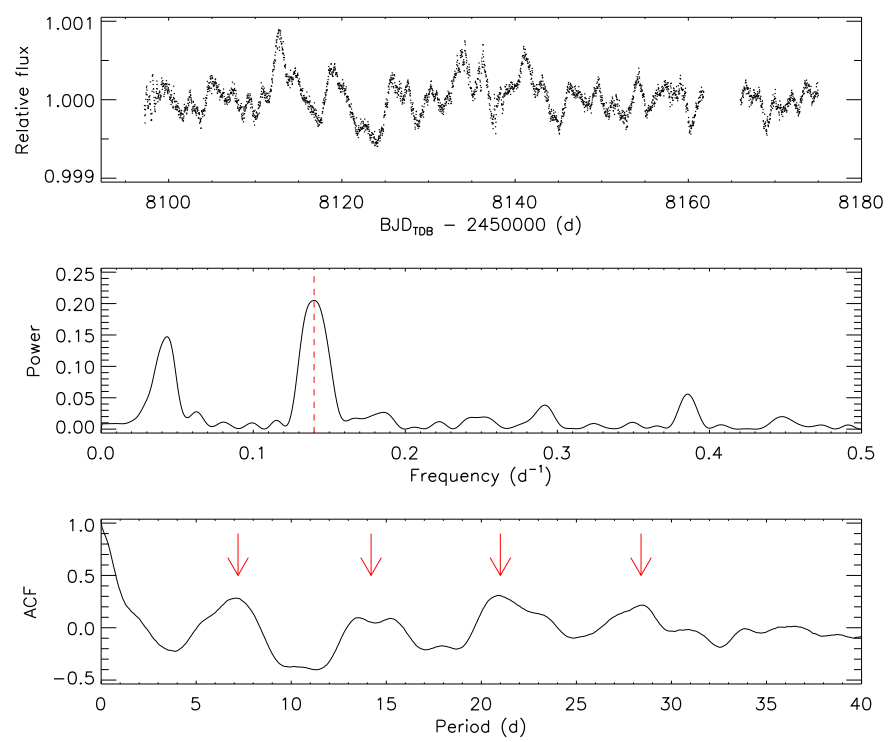

Fig. A.3. Upper panel: K2 light curve of EPIC 212036875 following the removal of the in-transit data points and the division by the best-fitting fourth-order cubic spline. Middle panel: GLS periodogram of the light curve. The red dashed line indicates the peaks at the rotation period of the star ( $\sim 7$ days). Lower panel: ACF of the light curve. The red arrows show the rotation period and its first three harmonics. 


\section{Appendix B: Formation by gravitational instability}

Given current uncertainties on the initial masses of fragments formed by gravitational instability and on their subsequent growth and migration (Kratter \& Lodato 2016; Fletcher et al. 2019), we evaluate the prospects for formation by disc instability using simple analytical prescriptions. We use the disc model of Ida et al. (2016), where the disc structure is determined by the viscosity, $\alpha$, and the mass flux through the disc, $\dot{M}_{\text {disc }}$. We evaluate at which radii it is Toomre unstable, and if so, whether the mass of EPIC $212036875 \mathrm{~b}$ is consistent with the expected fragment mass according to Eq. (49) in Kratter \& Lodato (2016). The fragment masses are shown in Fig. B.1. A self-gravitating disc maintains a viscosity $\alpha>0.01$, while Class I young stellar objects (YSOs) and FU Orionis stars (FUORs) have mass accretion rates up to a few times $10^{-5}$ (Robitaille et al. 2007; Gramajo et al. 2014). In these parameter ranges, our model forms fragments of several tens of Jupiter masses at $>10 \mathrm{AU}$, in agreement with previous works.

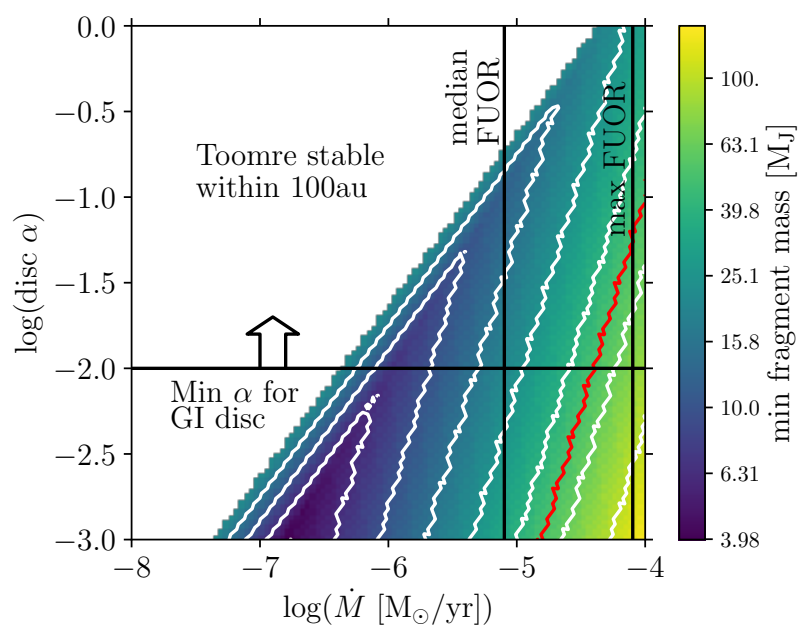

Fig. B.1. Formation of EPIC 212036875 b by gravitational instability in a protoplanetary disc. The contour plot shows the minimum mass of a fragment arising from disc instability as a function of the disc's viscosity and accretion rate. The red line indicates masses equal to the observed mass of EPIC 212036875 b. The horizontal black line shows the minimum $\alpha$ that a gravitationally unstable disc will generate, while the vertical black lines show the median and maximum accretion rates for FUOR discs found by Gramajo et al. (2014). Discs in the white region to the left are gravitationally stable, and hence do not form any fragments. 